\title{
The potassium paradox: Implications for soil fertility, crop production and human health
}

\author{
S.A. Khan*, R.L. Mulvaney and T.R. Ellsworth \\ Department of Natural Resources and Environmental Sciences, University of Illinois at Urbana-Champaign, \\ 1102 S. Goodwin Avenue, Urbana, IL 61801, USA. \\ *Corresponding author: potassiumparadox@gmail.com
}

Accepted 5 June 2013; First published online 10 October 2013

Research Paper

\begin{abstract}
Intensive fertilizer usage of $\mathrm{KCl}$ has been inculcated as a prerequisite for maximizing crop yield and quality, and relies on a soil test for exchangeable $\mathrm{K}$ in the plow layer to ensure that soil productivity will not be limited by nutrient depletion. The interpretive value of this soil test was rigorously evaluated by: (1) field sampling to quantify biweekly changes and seasonal trends, (2) characterizing the variability induced by air drying and the dynamic nature of soil $\mathrm{K}$ reserves and (3) calculating the $\mathrm{K}$ balance in numerous cropping experiments. These evaluations leave no alternative but to question the practical utility of soil $\mathrm{K}$ testing because test values cannot account for the highly dynamic interchange between exchangeable and non-exchangeable $\mathrm{K}$, exhibit serious temporal instability with or without air drying and do not differentiate soil $\mathrm{K}$ buildup from depletion. The need for routine $\mathrm{K}$ fertilization should also be questioned, considering the magnitude and inorganic occurrence of profile reserves, the recycling of $\mathrm{K}$ in crop residues and the preferential nature of $\mathrm{K}$ uptake. An extensive survey of more than 2100 yield response trials confirmed that $\mathrm{KCl}$ fertilization is unlikely to increase crop yield. Contrary to the inculcated perception of $\mathrm{KCl}$ as a qualitative commodity, more than 1400 field trials predominately documented a detrimental effect of this fertilizer on the quality of major food, feed and fiber crops, with serious implications for soil productivity and human health.
\end{abstract}

Key words: soil $\mathrm{K}$ testing, soil $\mathrm{K}$ reserves, residue $\mathrm{K}$ recycling, $\mathrm{KCl}$, potash fertilizer, agricultural sustainability, Cd bioaccumulation, Morrow Plots

\section{Introduction}

In the modern era of industrialized agriculture, there is a prevalent view of intensive fertilizer inputs as a prerequisite for maximizing yield, and hence short-term profitability. Unfortunately, several decades of these inputs can have unintended consequences for the chemical, physical and biological functioning of the soil resource, and for air, water and food quality ${ }^{1-3}$.

Implicit to intensive $\mathrm{K}$ fertilization is the bank account philosophy of managing soil fertility, which emphasizes the need for fertilizer inputs to at least replace crop removal ${ }^{4}$. According to this philosophy, a major rationale for $\mathrm{K}$ fertilization is to maintain soil reserves without regard to the economic importance of yield response ${ }^{5-7}$ or the overwhelming abundance of mineral $\mathrm{K}$ in most arable soils and subsoils, especially those dominated by 2:1 minerals $^{8-12}$. These reserves were recognized long ago for their fundamental role in supplying $\mathrm{K}$ for plant uptake, such that $\mathrm{K}$ fertilization was considered unnecessary when residues were returned to the soil ${ }^{8,9}$. With the entry of Canadian $\mathrm{KCl}$ into the world fertilizer market in the 1960s, the traditional approach to indigenous K fertility was displaced by growing reliance on a commercialized input.

Fertilizer K management originally utilized the sufficiency concept to predict yield response from exchangeable $\mathrm{K}$ in the plow layer ${ }^{13}$. An implicit assumption is that soil testing, typically carried out once in a 4-year interval, can adequately represent profile supplies of plant-available $\mathrm{K}$. This assumption, no less relevant to the basic cation saturation ratio concept ${ }^{14-16}$, is highly questionable in view of evidence that the exchangeable fraction is in a highly dynamic and temporally variable equilibrium with a vast storehouse of non-exchangeable and mineral $\mathrm{K}^{10,17-21}$ (see also supplemental references [1-3] for the online version of the paper). Since the 1970s, excessive $\mathrm{K}$ usage has been further intensified by the buildup-maintenance concept that inflates fertilizer consumption under the pretext of preventing yield reduction, and thus accentuates the economic interests of the fertilizer industry over those of the producer ${ }^{6,7}$. 
Under the latter concept, the sole purpose of soil $\mathrm{K}$ testing is to quantify fertilizer inputs for building up exchangeable $\mathrm{K}$ to a critical level that precludes the possibility of yield response, while still more $\mathrm{K}$ is prescribed as maintenance to replace annual crop $\mathrm{K}$ removal.

To ascertain whether the usual approach for exchangeable $\mathrm{K}$ testing, with or without air drying, provides a reliable basis for fertilizer $\mathrm{K}$ management, the work reported herein was conducted to quantify: (1) temporal and seasonal variability in $\mathrm{K}$ test values obtained through biweekly sampling of surface soil with no recent history of $\mathrm{KCl}$ fertilization; (2) $\mathrm{K}$ test changes induced by air drying soils that differed in long-term $\mathrm{KCl}$ inputs; (3) nonexchangeable $\mathrm{K}$ released by successive extraction; (4) K balance in relation to soil $\mathrm{K}$ test changes for cropping experiments with static fertilizer $\mathrm{K}$ inputs; and (5) the fertilizer value of $\mathrm{KCl}$ in a systematic survey of peerreviewed and university publications that encompass a global range of soil types, cropping practices and management systems in production settings. Based on these evaluations and the recycling of $\mathrm{K}$ from crop residues, we tested the null hypotheses that: (1) the usual approach to soil $\mathrm{K}$ testing is of no value for predicting fertilizer requirement or monitoring changes in K fertility, and (2) $\mathrm{KCl}$ fertilization will seldom lead to economic yield response or improve crop quality.

\section{Materials and Methods}

\section{Seasonality study}

To characterize seasonal changes in $\mathrm{K}$ test values, soil samples were collected at biweekly intervals between mid-March 1986 and 1990, from the University of Illinois South Farm at Urbana, IL. As detailed by $\mathrm{Khan}^{22}$, this sampling was done from six plots in a $\mathrm{K}$ rate study on a Drummer silty clay loam, classified as a fine-silty, mixed, superactive, mesic Typic Endoaquoll with montmorillonite and illite as the major 2:1 clay minerals ${ }^{23}$. The plots, each measuring $6 \mathrm{~m}$ wide and $24 \mathrm{~m}$ long with $<1 \%$ slope, had been used as a corn (Zea mays L.) breeding nursery cropped in alternate years to soybean (Glycine $\max \mathrm{L}$. Merr.), and had been fertilized annually with $\mathrm{KCl}$ from 1970 to 1983, at rates of $0,46,93,139,186$ and $232 \mathrm{~kg} \mathrm{Kha}^{-1}$. Of the six plots sampled by $\mathrm{Khan}^{22}$ for the seasonality study, the sole focus herein is on the plot that received no fertilizer $\mathrm{P}$ or $\mathrm{K}$, but only a spring application of $160-180 \mathrm{~kg} \mathrm{Nha}^{-1}$ as $\mathrm{NH}_{4} \mathrm{NO}_{3}$ when corn was grown between 1970 and 1990. Sampling followed a fixed protocol involving: (1) manual use of either a $2.5-\mathrm{cm}$ diameter probe or an auger; (2) care to avoid previous sampling points; and (3) collection of a five-core composite $(0-18 \mathrm{~cm})$ from each of three locations representing northern, central and southern subplots. The composite samples, in polyethylene bags, were immediately transported to the laboratory, passed through a $4.75 \mathrm{~mm}$ screen and thoroughly homogenized.
Triplicate 1.2-g subsamples were analyzed for fieldmoist exchangeable $\mathrm{K}$ following the ammonium acetate $\left(\mathrm{NH}_{4} \mathrm{C}_{2} \mathrm{H}_{3} \mathrm{O}_{2}\right)$-extraction technique described by Knudsen et al. ${ }^{24}$, using a Jenway Model PFP 7 flame photometer (Jenway, Essex, UK). So as to express these analyses on an air-dry basis, soil moisture content was determined by air drying two additional $1.2 \mathrm{~g}$ subsamples for $48 \mathrm{~h}$ in a forced-air oven at $40^{\circ} \mathrm{C}$. The remaining soil was transferred to a paper bag and subsequently air dried in the same oven for 10 days. The dried soil was crushed with a rolling pin to pass a $2 \mathrm{~mm}$ screen, mixed thoroughly in the bag and analyzed within 1 day for air-dried exchangeable $\mathrm{K}$.

\section{Drying study}

The effect of soil moisture level on the release of exchangeable $\mathrm{K}$ was investigated for the Drummer soil at Urbana where the seasonality study was conducted. After harvest in October 1989, a five-core composite was collected with a $2.5-\mathrm{cm}$ diameter soil probe to a depth of $18 \mathrm{~cm}$ at three locations within each of the four plots where the annual $\mathrm{K}$ rate had been $0-139 \mathrm{~kg} \mathrm{ha}^{-1}$, and the 15 cores collected from each plot were composited in polyethylene bags. The samples were transported to the laboratory, screened while field-moist to pass through a $4.75 \mathrm{~mm}$ sieve and spread to a depth of $2.5 \mathrm{~cm}$ on wooden trays. The trays were placed on laboratory benches for drying at approximately $20^{\circ} \mathrm{C}$ and $64 \%$ relative humidity, while air was continuously circulated across the samples that were periodically homogenized to ensure uniform moisture content. At 3-h intervals, triplicate samples from each tray were analyzed for moisture content and exchangeable $\mathrm{K}$ (expressed on an air-dried basis), until no further moisture loss was observed after $33 \mathrm{~h}$. These analyses were also performed on a portion of each airdried sample that had been oven-dried at $105^{\circ} \mathrm{C}$. The percentage increase in exchangeable $\mathrm{K}$ upon air drying was calculated as $100 \times\left(\mathrm{K}_{\mathrm{AD}}-\mathrm{K}_{\mathrm{FM}}\right) / \mathrm{K}_{\mathrm{FM}}$, where the field-moist $(\mathrm{FM})$ value was obtained at the initial soil moisture content and the air-dried (AD) value corresponds to a moisture content of approximately $40 \mathrm{~g} \mathrm{~kg}^{-1}$.

\section{Speciation study}

Biweekly soil samples $(0-18 \mathrm{~cm})$ collected and air dried for the seasonality study in August and September were composited each year between 1986 and 1989 to represent the plot that had received $\mathrm{N}$ but no $\mathrm{P}$ or $\mathrm{K}$ fertilization

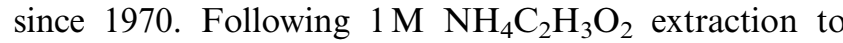
determine exchangeable $\mathrm{K}$, six successive digestions were performed to liberate non-exchangeable $\mathrm{K}$ with boiling $1 \mathrm{M} \mathrm{HNO}_{3}{ }^{24}$. The digests were centrifuged, and the supernatant was analyzed for $\mathrm{K}$ by flame photometry as described previously.

Total K analyses were performed on samples composited from 1986 and 1989, by fusion of an oven-dried $\left(105^{\circ} \mathrm{C}\right)$ portion with $\mathrm{LiBO}_{2} \cdot 8 \mathrm{H}_{2} \mathrm{O}$ in a $\mathrm{Pt}$ crucible ${ }^{25}$. 
The fused sample was dissolved in $0.04 \mathrm{M} \mathrm{HNO}_{3}$ and analyzed by atomic emission spectroscopy using a Perkin Elmer Model 3110 spectrometer (Perkin Elmer, Norwalk, CT) and an air- $\mathrm{C}_{2} \mathrm{H}_{2}$ flame.

\section{Morrow Plots potassium balance study}

To ascertain whether the conventional approach to soil $\mathrm{K}$ testing reflects net $\mathrm{K}$ balance, exchangeable $\mathrm{K}$ was determined for surface $(0-15 \mathrm{~cm})$ samples collected in 1955 and 2005 from the Morrow Plots, where the world's longest continuous cropping experiment on the most productive soil order (Mollisols) has been conducted since 1876. The samples used in our work represent six unreplicated subplots currently designated as 3NA and $3 \mathrm{NB}$ under continuous corn, $4 \mathrm{NA}$ and $4 \mathrm{NB}$ under a 2-year rotation of corn and oats (Avena sativa L.) or soybean (since 1967) and 5NA and 5NB under a 3-year rotation of corn, oats and alfalfa (Medicago sativa $\mathrm{L}$.) hay, where B- but not A-series subplots receive NPK fertilization that supplied $28-186 \mathrm{~kg} \mathrm{Kha}^{-1}$ in some but not all years. The soil is classified as Flanagan silt loam, a fine, smectitic, mesic Aquic Argiudoll with illite and smectite as the dominant 2:1 clay minerals ${ }^{26}$. Further details concerning the Morrow Plots and their management can be found in Khan et al. ${ }^{27}$.

A baseline for the study period was established using air-dried soil samples collected in the spring of 1955 prior to any application of $\mathrm{KCl}$, which were obtained from an archival collection maintained by the University of Illinois in individually labeled air-tight glass containers. The 2005 samples consisted of triplicate soil cores that were collected just after harvest, air dried at $40^{\circ} \mathrm{C}$ and screened to $<2 \mathrm{~mm}$. Analyses for exchangeable $\mathrm{K}$ were performed in triplicate as described previously, by processing all samples in a single batch. To allow expression on a mass basis $\left(\mathrm{kgha}^{-1}\right)$, test values were multiplied by the corresponding bulk density, obtained from direct measurements in 2005 , or by using pedotransfer functions to estimate 1955 values. A more detailed description is available in Khan et al. ${ }^{27}$.

A K balance was constructed for 1955-2005, based on cumulative fertilizer input and crop removal. Morrow Plot records were utilized to document $\mathrm{KCl}$ inputs, whereas crop $\mathrm{K}$ removal was estimated using composition data from the Illinois Agronomy Handbook ${ }^{28}$ in conjunction with yield and management records. The handbook values used for crop $\mathrm{K}$ composition are typical of university maintenance recommendations to replace $\mathrm{K}$ removed in alfalfa hay $\left(20.8 \mathrm{~kg} \mathrm{Mg}^{-1}\right)$ or by corn $\left(4.2 \mathrm{~kg} \mathrm{Mg}^{-1}\right)$, soybean $\left(18.0 \mathrm{~kg} \mathrm{Mg}^{-1}\right)$ and oats $\left(5.2 \mathrm{~kg} \mathrm{Mg}^{-1}\right)$ harvested for grain. The yield records used were expressed as dry tons acre ${ }^{-1}$ for alfalfa, or as bushels acre ${ }^{-1}$ at a market-standard moisture content of $155 \mathrm{~g} \mathrm{~kg}^{-1}(15.5 \%)$ for corn (56 lb bushel $\left.{ }^{-1}\right), 130 \mathrm{~g} \mathrm{~kg}^{-1}$ $(13 \%)$ for soybean $\left(60 \mathrm{lb} b u s h e l^{-1}\right)$ and $140 \mathrm{~g} \mathrm{~kg}^{-1}(14 \%)$

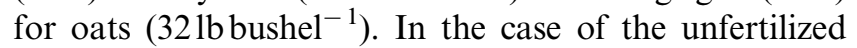

(NA-series) subplots, crop K removal was intensified by the harvest of corn or oats stover until 1967, estimated at $331 \mathrm{~kg} \mathrm{Kha}^{-1}$ for continuous corn, $310 \mathrm{~kg} \mathrm{Kha}^{-1}$ for the corn-oats rotation, and $256 \mathrm{~kg} \mathrm{~K} \mathrm{ha}^{-1}$ for the corn-oatshay rotation. To account for $\mathrm{K}$ removal in these residues, a harvest index of 0.5 was assumed for both crops $^{29-32}$, and a $\mathrm{K}$ concentration of 13.5 (corn) or 15 (oats) $\mathrm{kg} \mathrm{Mg}^{-1}$ of dry matter ${ }^{33}$.

\section{Potassium balance for other studies}

In a more comprehensive evaluation of exchangeable $\mathrm{K}$ testing for air-dried soil, a systematic review of the literature was performed to compile a global database of short- and long-term $\mathrm{K}$ response experiments involving fixed (static) fertilizer treatments and representing a wide range in soils, cropping and management practices. This review utilized the $\mathrm{CAB}$ citation index and reviews of relevant individual journals. Three attributes were critical in selecting these data sets: (1) soil test values for exchangeable $\mathrm{K}$ determined at the beginning and end of the study period; (2) fertilizer $\mathrm{K}$ inputs throughout the study period; and (3) yield or $\mathrm{K}$ removal data for all crop material harvested. In cases where soil $\mathrm{K}$ test data were reported as a mass-based concentration, a conversion was made to $\mathrm{kg} \mathrm{ha}^{-1}$ assuming a bulk density of $1.47 \mathrm{Mg} \mathrm{m}^{-3}$, which corresponds to the conventional weight of 2 million pounds $(907 \mathrm{Mg})$ per acre $(0.405 \mathrm{ha})$ for a plow layer 6 inches $(15 \mathrm{~cm})$ deep. When necessary, crop $\mathrm{K}$ removal was calculated from yield data by utilizing published $\mathrm{K}$ concentrations for grain ${ }^{28}$ and/or stover $^{33}$.

\section{Fertilizer value of potassium chloride}

To ascertain the benefits of $\mathrm{KCl}$ fertilization for crop yield and quality, an extensive effort was made in surveying peer-reviewed and university data from published field studies not exceeding 10 years in duration. For the yield survey, 211 publications reporting statistical analyses of yield differences were utilized in calculating percentage response to $\mathrm{KCl}$ fertilization as $100 \times$ (fertilized yield unfertilized yield)/unfertilized yield. Qualitative impacts of this fertilizer were evaluated as positive, negative, none or variable, according to what was reported in 139 publications comparing a specific parameter of crop quality with and without the use of $\mathrm{KCl}$. Major emphasis was placed on broad coverage of the scientific literature, encompassing food, feed and fiber crops grown under various management practices on a wide range of soil types throughout the world.

An effort was also made in utilizing the scientific literature to assess the impact of $\mathrm{KCl}$ fertilization on two key aspects of soil productivity: cation-exchange capacity (CEC) and microbial $\mathrm{N}$ cycling. Interest in these topics was motivated by $\mathrm{K}^{+}$fixation that promotes interlayer collapse of 2:1 clay minerals, and by the inhibitory effects of $\mathrm{KCl}$ on biological $\mathrm{N}_{2}$ fixation and nitrification. 


\section{Statistical analyses}

Seasonality and linear trend analysis of the 4-year time series of air-dried test levels of exchangeable $\mathrm{K}\left(\mathrm{kgha}^{-1}\right)$ was evaluated statistically as described by Lovell ${ }^{34}$. Paired $t$-tests were performed on samples collected post-harvest in October 1989 to monitor changes in soil test K upon air drying. Exchangeable $\mathrm{K}$ data obtained for the Morrow Plots were analyzed statistically with PROC MIXED in SAS $^{35}$, such that the 2005 data provided a variance estimate for the standard error of mean values obtained as a five-core composite in $1955^{27}$. The step-down Bonferroni adjustment of $P$ values ${ }^{36}$ was performed with PROC MULTEST in $\mathrm{SAS}^{35}$ for multiple comparison tests to evaluate net changes in exchangeable $\mathrm{K}$ between 1955 and 2005.

\section{Results and Discussion}

\section{Evaluation of soil potassium testing}

Soil testing is widely regarded as the best approach for making economical fertilizer recommendations or monitoring soil fertility changes in relation to management practices. If these objectives are to be properly met, soil sampling must be adequate to represent the field area under investigation, and test values should be calibrated to crop response and sufficiently stable that interpretations are unaffected by the time of sampling or the method of sample processing.

In the modern era of input-intensive agriculture, soil testing is often done to measure exchangeable $\mathrm{K}$, following the concept originated by Bray ${ }^{13}$. This prevalence reflects the major emphasis given to rapid methods of sample processing and analysis in commercial soil testing, despite an inherent complication that has long been recognized: $\mathrm{K}$ test values can change markedly when samples are air dried to expedite processing ${ }^{37,38}$ (see also supplemental references [4-8] for the online version of the paper). A further complication arises because of variability associated with the time of sampling ${ }^{18,21}$ (see also supplemental references [4] and [9] for the online version of the paper), which becomes a major source of error in soil testing for site-specific management ${ }^{39}$.

Both factors clearly contributed to the variability documented in Fig. 1 for a 4-year field study that involved biweekly sampling to monitor changes in exchangeable $\mathrm{K}$ test values with and without air drying. Any such changes would have been minimized because the work involved a single investigator utilizing a fixed protocol and experimental design, and a study site devoid of fertilizer $\mathrm{K}$ inputs for at least 14 years. By repeatedly imposing a dense sampling design on a single small research plot subject to minimal compaction from mechanical traffic, spatial variability was minimized so as to better quantify any occurrence of temporal variability.

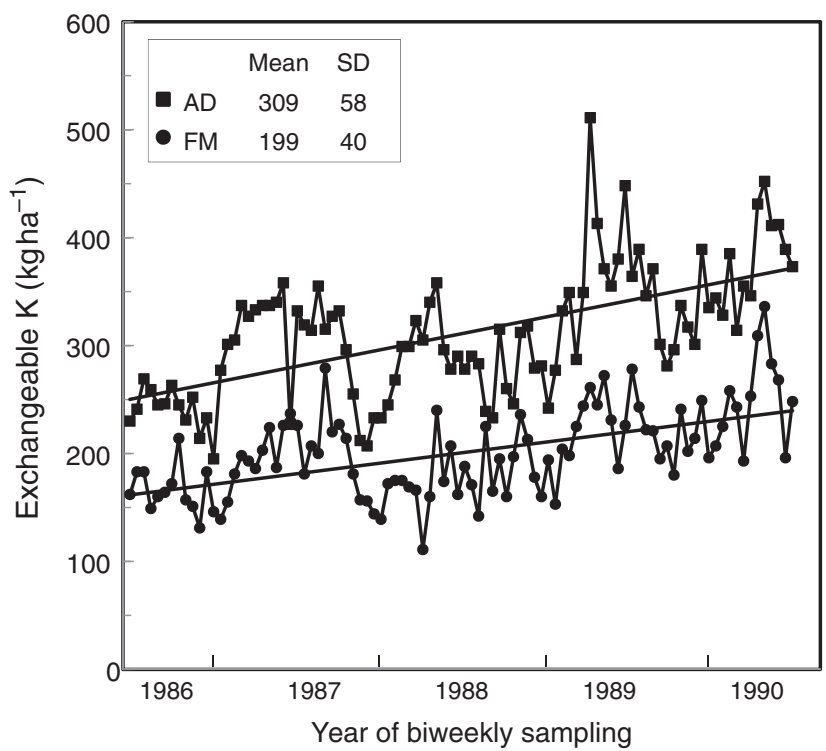

Figure 1. Biweekly changes in exchangeable $\mathrm{K}$ for field-moist (FM) and air-dried (AD) samples $(0-18 \mathrm{~cm})$ collected on 96 dates between March 13, 1986 and March 15, 1990, from a Drummer soil under a corn (Zea mays L.)-soybean (Glycine $\max \mathrm{L}$. Merr.) rotation. No fertilizer $\mathrm{P}$ or $\mathrm{K}$ had been applied since 1970. Data points are a mean of nine determinations representing triplicate analyses performed on five-core composites collected from each of three subplots. Trendlines were generated by linear regression. SD, standard deviation.

Despite these precautions, Fig. 1 shows that soil $\mathrm{K}$ test levels, with or without air drying, varied drastically with the biweekly sampling strategy adopted throughout the study period, and the variation became more extensive as test values began to increase after harvest, during the usual sampling period for commercial soil testing in a temperate region such as the Midwestern USA. Statistical analyses were highly significant $(P<0.001)$ in detecting a seasonality effect on exchangeable soil $\mathrm{K}$, which is consistent with several previous field studies ${ }^{40,41}$ (see also supplemental references [10-13] for the online version of the paper) that document greater test values during the winter months. This increase can be attributed to a high soil moisture content that promotes the leaching of $\mathrm{K}$ from crop residues and the conversion of nonexchangeable to exchangeable $\mathrm{K}$ through valence dilution. After seasonality adjustment, a highly significant $(P<0.001)$ linear increase in exchangeable $\mathrm{K}$ was observed over the 4-year study period.

Drying was expected to decrease temporal variability by homogenizing soil samples; however, the data in Fig. 1 reveal no such effect as the coefficient of variation was nearly the same before and after air drying that escalated $\mathrm{K}$ test values by $55 \%$ on average. A major complication can also be found in the numerous incoherencies between field-moist and air-dried test values documented by Fig. 1, which precludes the use of a simple correction factor to compensate for air drying or the timing of sample 


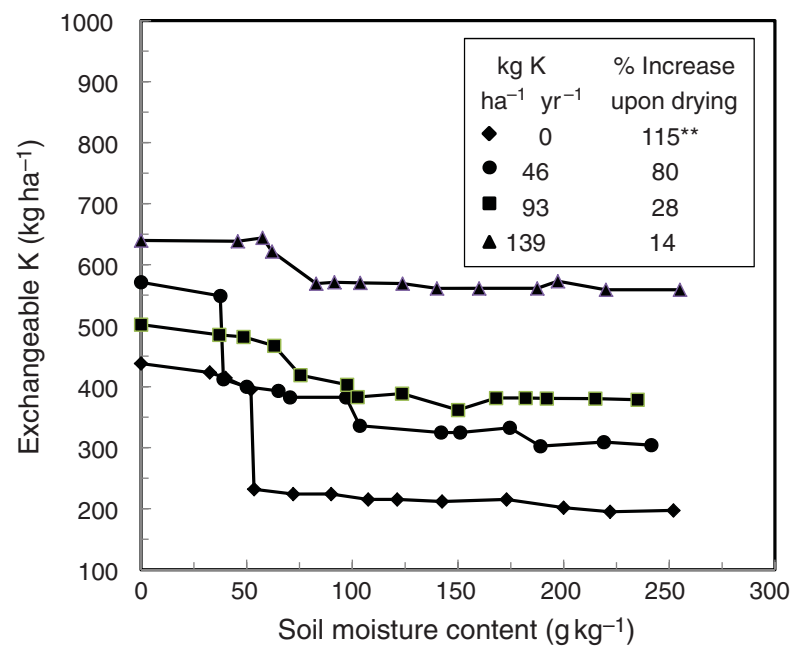

Figure 2. Potassium release curves for a Drummer soil cropped to a corn (Zea mays L.)-soybean (Glycine max L. Merr.) rotation, with or without annual application of 46$139 \mathrm{~kg} \mathrm{~K} \mathrm{ha}^{-1}$ as $\mathrm{KCl}$. Field-moist samples $(0-18 \mathrm{~cm})$ were air dried to approximately $40 \mathrm{~g} \mathrm{~kg}^{-1}$ in a forced-air oven at $40^{\circ} \mathrm{C}$, followed by oven-drying at $105^{\circ} \mathrm{C}$. Data points are a mean of triplicate determinations performed on five-core composites collected from each of three subplots. Error bars representing one standard deviation are shown, when they exceed the size of the data marker, for field-moist (FM) and air-dried (AD) test values. The percentage increase upon air drying was calculated as $100 \times\left(\mathrm{K}_{\mathrm{AD}}-\mathrm{K}_{\mathrm{FM}}\right) / \mathrm{K}_{\mathrm{FM}}$. **, significant at $P<0.01$.

collection. Both findings raise a serious concern that soil $\mathrm{K}$ testing by the usual approach is of no practical value, in contrast to the advocacy that is common in extension publications and presentations, trade magazines and popular articles ${ }^{28,42}$.

The same concern emerged when sample drying was evaluated by a one-time sampling of soils that ranged widely in $\mathrm{K}$ fertility, so as to eliminate the confounding effect of seasonality. The results (Fig. 2) follow the usual trend toward higher test values upon air drying; however, the increase tended to be greater as the fertility level decreased, further demonstrating the difficulties inherent in correcting air-dried test values to a field-moist basis. This finding reveals that air-dried $\mathrm{K}$ test values can easily be misinterpreted when utilized for predicting $\mathrm{K}$ fertilizer requirement under low fertility management or for monitoring buildup/depletion.

A more rigorous evaluation becomes possible when soil $\mathrm{K}$ test data span a prolonged period documented with a complete record of $\mathrm{K}$ inputs and outputs. The Morrow Plots serve as an ideal resource for this purpose, providing three cropping systems that include the corn-soybean rotation common throughout the Midwestern USA. With this rotation, and also with continuous corn, $\mathrm{K}$ removal from the Morrow Plots occurs only in grain, whereas the intensifying effect of forage production is present with a 3-year rotation of corn, oats and alfalfa hay.
As shown by Table $1, \mathrm{~K}$ test levels for the surface soil were invariably increased by 51 years of cropping. Remarkably, the largest increases, highly significant at $P<0.001$, occurred in the absence of NPK fertilization, when the $\mathrm{K}$ balance was decidedly negative for continuous corn or the corn-oats-hay rotation. Equally remarkable is the substantial soil test increase observed for the unfertilized subplot in the two-crop rotation, despite $\mathrm{K}$ removal that totaled $1.4 \mathrm{Mg} \mathrm{ha}^{-1}$. These observations are consistent with the seasonality study discussed previously (Fig. 1), which showed an upward trend over time in airdried and field-moist $\mathrm{K}$ test levels in the absence of fertilizer $\mathrm{K}$ inputs. No less troubling are the similar net changes documented by this soil test for the three subplots receiving NPK, regardless of whether fertilizer K inputs were above (continuous corn or corn-oats-hay) or below (corn-soybean) crop removal. The implication is that the $\mathrm{K}$ test cannot differentiate soil $\mathrm{K}$ buildup from depletion.

The latter flaw is also apparent from the sheer magnitude of the $\mathrm{K}$ test levels reported in Table 1 for the three unfertilized subplots, particularly those under continuous corn and the corn-oats-hay rotation. By 2005, following 130 years of $\mathrm{K}$ removal, test values for these subplots were within the range of critical levels calibrated for North America ${ }^{43}$, which would normally be interpreted as evidence of successful fertilizer K management.

To ascertain whether soil $\mathrm{K}$ testing is generally subject to the aforementioned difficulties, baseline changes in exchangeable $\mathrm{K}$ were compared with cumulative $\mathrm{K}$ balance compiled for numerous field experiments with and without $\mathrm{K}$ fertilization, encompassing a global range of soil orders, cropping systems and management practices. The resulting database (Table 2), consisting of 68 trials, provides no convincing evidence that soil test $\mathrm{K}$ reflects the net balance of $\mathrm{K}$ addition and removal. The most disturbing disparities involved 17 trials in which test levels were either constant or increased while crop $\mathrm{K}$ removal far exceeded fertilizer inputs or occurred in the complete absence of fertilization. Such obvious incongruities, paralleling what was found for the Morrow Plots (Table 1), leave little alternative but to question the validity of soil testing for exchangeable $\mathrm{K}$, and this concern is especially relevant to the developing world, where soil $\mathrm{K}$ removal has been intensified by many centuries of grain and biomass harvest. To make matters worse, Table 2 shows that $\mathrm{K}$ test values sometimes decreased with a positive $\mathrm{K}$ balance, which according to the usual interpretation would justify unnecessary $\mathrm{K}$ fertilization. The implication is that soil $\mathrm{K}$ testing does not provide a scientific basis for fertilizer $\mathrm{K}$ management.

This dilemma is inherent to a one-time measurement of exchangeable $\mathrm{K}$, which can never represent the highly dynamic effect of soil moisture on the interchange with vastly larger soil pools that occur in the form of nonexchangeable and mineral $\mathrm{K}^{80}$. The dynamic nature of soil $\mathrm{K}$ is no less relevant in refuting the central assumption implicit to soil $\mathrm{K}$ testing, namely, that plant $\mathrm{K}$ availability 
Table 1. Soil potassium test changes and potassium balance for the Morrow Plots, 1955-2005.

\begin{tabular}{|c|c|c|c|c|c|c|c|}
\hline \multirow[b]{2}{*}{ Rotation $^{I}$} & \multirow{2}{*}{$\begin{array}{l}\text { Fertilizer } \\
\text { treatment }^{2}\end{array}$} & \multicolumn{3}{|c|}{ Exchangeable $\mathbf{K}^{3}$} & \multicolumn{3}{|c|}{ Cumulative } \\
\hline & & Initial $^{4}$ & Final $^{5}$ & Net change & $\mathrm{K}$ added & $K$ removed & Net $\mathrm{K}$ input \\
\hline & & & & $\ldots$ & 1 & & - - - - - - \\
\hline \multirow[t]{2}{*}{$\mathrm{C}-\mathrm{C}$} & None & $242(4)$ & $403(33)$ & $161 * * *$ & 0 & 892 & -892 \\
\hline & NPK & $242(4)$ & $354(22)$ & $112 * * *$ & 2231 & 1692 & 539 \\
\hline \multirow[t]{2}{*}{$\mathrm{C}-\mathrm{O}(\mathrm{S})$} & None & $218(4)$ & $304(25)$ & $86^{* * *}$ & 0 & $1706^{6}$ & -1706 \\
\hline & NPK & $218(4)$ & $327(26)$ & $109 * * *$ & 1934 & $2136^{7}$ & -202 \\
\hline \multirow[t]{2}{*}{$\mathrm{C}-\mathrm{O}-\mathrm{H}$} & None & $217(6)$ & $382(18)$ & $165 * * *$ & 0 & $1088^{8}$ & -1088 \\
\hline & NPK & $217(6)$ & $332(27)$ & $115^{* * *}$ & 3114 & $2865^{9}$ & 249 \\
\hline
\end{tabular}

*** Significant at $\alpha=0.001$ by the step-down Bonferroni procedure.

${ }^{1}$ C, corn (Zea mays L.); H, alfalfa (Medicago sativa L.) hay; O, oats (Avena sativa L.); S, soybean (Glycine max L. Merr.). Since 1967 , the two-crop rotation has involved soybean instead of oats.

${ }^{2}$ NPK, nitrogen-phosphorus-potassium fertilization using urea (168 [1955-1966] or 224 [since 1967] $\mathrm{kg} \mathrm{Nha}^{-1} \mathrm{yr}^{-1} \mathrm{for} \mathrm{corn}$, $28 \mathrm{~kg} \mathrm{Nha}^{-1} \mathrm{yr}^{-1}$ for oats), triple superphosphate $\left(0-96 \mathrm{kgPha}^{-1} \mathrm{yr}^{-1}\right)$ and $\mathrm{KCl}\left(0-186 \mathrm{~kg} \mathrm{Kha}^{-1} \mathrm{yr}^{-1}\right)$. No amendment was applied before 1955 .

${ }_{3}$ Determined by $\mathrm{NH}_{4} \mathrm{C}_{2} \mathrm{H}_{3} \mathrm{O}_{2}$ extraction ${ }^{24}$ of air-dried samples.

${ }^{4}$ Mean values reported from triplicate determinations of a single archived five-core composite soil sample $(0-15 \mathrm{~cm})$. Standard deviations shown in parentheses.

5 Mean values reported from triplicate determinations performed on each of three replicate soil samples $(0-15 \mathrm{~cm})$. Standard deviations shown in parentheses.

${ }^{6}$ Estimated as $44 \%$ by corn, $7 \%$ by oats and $49 \%$ by soybean.

7 Estimated as $49 \%$ by corn, $3 \%$ by oats and $48 \%$ by soybean.

8 Estimated as $54 \%$ by corn, $25 \%$ by oats and $21 \%$ by alfalfa hay.

9 Estimated as $26 \%$ by corn, $65 \%$ by oats and $9 \%$ by alfalfa hay.

is directly related to exchangeable $\mathrm{K}$ in the surface soil. Table 2 leaves no doubt that plant $\mathrm{K}$ uptake must originate from other sources, and is consistent with previous evidence that soil $\mathrm{K}$ reserves contribute considerable quantities of plant-available $\mathrm{K}^{10,11,20,75,78,81-83}$ (see also supplemental references [14-26] for the online version of the paper).

These reserves were once recognized to be a major source of K-supplying power for the highly productive soils that dominate the Corn Belt, as well as for most other areas of the continental USA ${ }^{8,9,84}$. The importance of the non-exchangeable fraction as a storehouse for exchangeable and soluble $\mathrm{K}$ is documented by Table 3 , which shows the magnitude of this fraction by six successive determinations performed on surface samples of a soil cropped for 17-20 years with no K input. Initial recoveries were three- to fivefold higher for non-exchangeable than exchangeable $\mathrm{K}$, followed by a gradual decline toward a stable level of $240-300 \mathrm{~kg} \mathrm{ha}^{-1}$, with approximately $3000 \mathrm{kgha}^{-1}$ as the cumulative recovery of non-exchangeable $\mathrm{K}$. The resilient behavior of soil $\mathrm{K}$ is further revealed, in that 4 years of crop $\mathrm{K}$ removal had no consistent effect on soil concentrations of exchangeable, non-exchangeable or total $\mathrm{K}$, implicating the mineral fraction as an important source of buffering. These findings are to be expected, considering what has long been known about the availability and dynamics of non-exchangeable and mineral $\mathrm{K}$, based on chemical extraction $^{19,85-87}$ (see also supplemental references [22] and [27-30] for the online version of the paper), exhaustive cropping $^{81,86,88,89}$ (see supplemental references [8], [15] and [16] for the online version of the paper) and electrodialysis ${ }^{83,90}$

The importance of non-exchangeable and mineral $\mathrm{K}$ for plant uptake is by no means unique to micaceous clays, which are subject to biological weathering in the rhizosphere ${ }^{91-94}$ (see also supplemental references [28] and [31] for the online version of the paper). Sand- and siltsized muscovite and biotite can also be a major source of plant-available $\mathrm{K}$, as demonstrated conclusively by Mengel et al. ${ }^{83}$ in studies with 14 loess-derived Alfisols. These primary minerals, and also $\mathrm{K}$ feldspars, are believed to account for the high K-supplying power of sandy Ultisols of the Atlantic Coastal Plain ${ }^{82,95-97}$, while nonexchangeable $\mathrm{K}$ reserves can even be important for growing a shallow-rooted crop such as pineapple on the basaltic Andisols of Hawaii ${ }^{81}$.

Soil $\mathrm{K}$ reserves are much greater for the profile than the plow layer ${ }^{12,62,95,97,98}$ (see also supplemental references [32-34] for the online version of the paper), and can be more fully exploited by many agricultural crops with a well-developed rooting system. The importance of subsoil $\mathrm{K}$ is clearly apparent from $\mathrm{K} / \mathrm{Rb}$ isotope dilution studies demonstrating that the extent of uptake is directly proportional to rooting depth ${ }^{99-101}$. This uptake is largely concentrated in the vegetative biomass $^{33,102}$, and enriches the surface soil when inorganic $\mathrm{K}$ leaches from plant shoots or residues ${ }^{8,9,81,102-106}$ (see supplemental references [35-45] for the online version of the paper). 
Table 2. Soil potassium test changes in relation to potassium balance, as reported for static plot experiments.

\begin{tabular}{|c|c|c|c|c|c|c|c|c|c|c|c|}
\hline \multirow[b]{2}{*}{ Location } & \multirow[b]{2}{*}{ Soil order ${ }^{1}$} & \multirow{2}{*}{$\begin{array}{l}\text { Cropping } \\
\text { system }^{2}\end{array}$} & \multirow{2}{*}{$\begin{array}{l}\text { Study } \\
\text { period }\end{array}$} & \multirow{2}{*}{$\begin{array}{c}\text { Sampling } \\
\text { depth }\end{array}$} & \multicolumn{3}{|c|}{${\text { Exchangeable } \mathbf{K}^{3}}^{3}$} & \multicolumn{3}{|c|}{ Cumulative } & \multirow[b]{2}{*}{ Reference(s) } \\
\hline & & & & & Initial & Final & Net change & K added & $\mathrm{K}$ removed & Net $K$ input & \\
\hline & & & & $\mathrm{cm}$ & \multicolumn{6}{|c|}{ 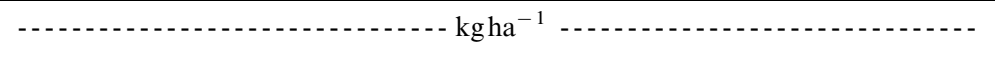 } & \\
\hline $\begin{array}{l}\text { USA } \\
\text { Alabama }\end{array}$ & $\begin{array}{l}\text { Ultisols } \\
\quad\left(\mathrm{fsl}_{4}, \mathrm{scl}_{2}, \mathrm{sil}_{1}\right)\end{array}$ & $\mathrm{C}-\mathrm{Ct}(7)$ & $1954-1969$ & $0-15$ & 113 & 104 & -9 & 0 & 332 & -332 & $44-46$ \\
\hline \multirow{5}{*}{ Illinois } & & & & & 113 & 229 & 115 & 1488 & 513 & 975 & \\
\hline & Mollisols (sil) & $\mathrm{C}-\mathrm{S}(2)$ & $1982-1987$ & $0-18$ & $\begin{array}{l}322 \\
322\end{array}$ & $\begin{array}{l}335 \\
476\end{array}$ & $\begin{array}{r}13 \\
154\end{array}$ & $\begin{array}{r}0 \\
334\end{array}$ & $\begin{array}{l}290 \\
325\end{array}$ & $\begin{array}{r}-290 \\
9\end{array}$ & 47 \\
\hline & Entisols (sil) & $\mathrm{C}-\mathrm{S}$ & 1994-1996 & $0-20$ & 288 & 253 & -35 & 0 & 325 & -325 & 48 \\
\hline & & & & & 316 & 317 & 1 & 335 & 330 & 5 & \\
\hline & Mollisols (sil) & $\mathrm{C}-\mathrm{S}$ & 1994-1996 & $0-20$ & $\begin{array}{l}280 \\
314\end{array}$ & $\begin{array}{l}283 \\
339\end{array}$ & $\begin{array}{r}3 \\
26\end{array}$ & $\begin{array}{r}0 \\
335\end{array}$ & $\begin{array}{l}270 \\
282\end{array}$ & $\begin{array}{r}-270 \\
52\end{array}$ & \\
\hline Indiana & Mollisols (sil) & $\mathrm{C}-\mathrm{S}-\mathrm{W}_{\mathrm{w}}-\mathrm{C}$ & $1964-1983$ & $0-20$ & $\begin{array}{r}93 \\
178\end{array}$ & $\begin{array}{l}112 \\
422\end{array}$ & $\begin{array}{r}19 \\
243\end{array}$ & $\begin{array}{r}0 \\
2790\end{array}$ & $\begin{array}{l}506 \\
723\end{array}$ & $\begin{array}{r}-506 \\
2067\end{array}$ & 49 \\
\hline \multirow[t]{2}{*}{ Iowa } & Mollisols (cl) & $\mathrm{C}-\mathrm{S}$ & 1976-1989 & $0-15$ & $\begin{array}{l}10(3) \\
10(3)\end{array}$ & $\begin{array}{l}10(3) \\
19(7)\end{array}$ & $\begin{array}{r}(0) \\
9(4)\end{array}$ & $\begin{array}{r}0 \\
1207\end{array}$ & $\begin{array}{l}501 \\
600\end{array}$ & $\begin{array}{r}-501 \\
607\end{array}$ & 50 \\
\hline & Mollisols (1) & $\mathrm{C}-\mathrm{S}$ & 1979-1989 & $0-15$ & $\begin{array}{l}40(4) \\
37(2)\end{array}$ & $\begin{array}{l}17(7) \\
61(4)\end{array}$ & $\begin{array}{r}-22(7) \\
24(2)\end{array}$ & $\begin{array}{r}0 \\
1478\end{array}$ & $\begin{array}{l}474 \\
481\end{array}$ & $\begin{array}{r}-474 \\
998\end{array}$ & 51 \\
\hline Michigan & Alfisols (ls) & $\mathrm{C}-\mathrm{C}$ & 1974-1982 & $0-22$ & $\begin{array}{l}266 \\
601\end{array}$ & $\begin{array}{l}260 \\
598\end{array}$ & $\begin{array}{r}-6 \\
-3\end{array}$ & $\begin{array}{r}335 \\
1595\end{array}$ & $\begin{array}{l}284 \\
276\end{array}$ & $\begin{array}{r}51 \\
1319\end{array}$ & 52 \\
\hline Minnesota & Mollisols (cl) & $\mathrm{C}-\mathrm{C}(2)$ & 1974-1981 & $0-20$ & $\begin{array}{l}227 \\
237\end{array}$ & $\begin{array}{l}302 \\
315\end{array}$ & $\begin{array}{l}75 \\
78\end{array}$ & $\begin{array}{r}0 \\
372\end{array}$ & $\begin{array}{l}258 \\
267\end{array}$ & $\begin{array}{r}-258 \\
105\end{array}$ & 53,54 \\
\hline Missouri & Alfisols (sil) & $\begin{array}{l}\mathrm{W}_{\mathrm{s}}-\mathrm{W}_{\mathrm{s}} \\
\mathrm{C}-\mathrm{C}\end{array}$ & $\begin{array}{l}1963-1989 \\
1963-1989\end{array}$ & $\begin{array}{l}0-20 \\
0-20\end{array}$ & $\begin{array}{l}400 \\
414\end{array}$ & $\begin{array}{l}303 \\
364\end{array}$ & $\begin{array}{r}-97 \\
50\end{array}$ & $\begin{array}{l}0 \\
0\end{array}$ & $\begin{array}{l}87 \\
50\end{array}$ & $\begin{array}{l}-87 \\
-50\end{array}$ & 55 \\
\hline Nebraska & Mollisols (sicl) & $\mathrm{C}-\mathrm{C}$ & 1973-1984 & $0-15$ & $\begin{array}{l}598 \\
598\end{array}$ & $\begin{array}{l}77(3) \\
83(1)\end{array}$ & $\begin{array}{l}17(5) \\
23(3)\end{array}$ & $\begin{array}{l}156 \\
720\end{array}$ & $\begin{array}{l}471 \\
486\end{array}$ & $\begin{array}{r}-315 \\
234\end{array}$ & 6,56 \\
\hline New Jersey & Ultisols (1) & $\mathrm{A}-\mathrm{A}$ & $1953-1961$ & $0-30$ & $\begin{array}{l}131 \\
222\end{array}$ & $\begin{array}{l}105 \\
660\end{array}$ & $\begin{array}{r}26 \\
438\end{array}$ & $\begin{array}{r}0 \\
3357\end{array}$ & $\begin{array}{r}412 \\
2006\end{array}$ & $\begin{array}{r}-412 \\
1351\end{array}$ & 57 \\
\hline North Carolina & Ultisols (s) & $\mathrm{Cb}-\mathrm{Cb}$ & $1954-1965$ & $0-15$ & $\begin{array}{l}53 \\
53\end{array}$ & $\begin{array}{r}9 \\
53\end{array}$ & $\begin{array}{r}-44 \\
0\end{array}$ & $\begin{array}{r}0 \\
2231\end{array}$ & $\begin{array}{r}215 \\
2202\end{array}$ & $\begin{array}{r}-215 \\
29\end{array}$ & 58 \\
\hline Oklahoma & Mollisols (1) & $\mathrm{W}_{\mathrm{w}}-\mathrm{W}_{\mathrm{w}}$ & 1938-2001 & $0-15$ & $\begin{array}{l}508 \\
464\end{array}$ & $\begin{array}{l}414 \\
548\end{array}$ & $\begin{array}{r}-94 \\
84\end{array}$ & $\begin{array}{r}0 \\
1843\end{array}$ & $\begin{array}{l}1219 \\
1201\end{array}$ & $\begin{array}{r}-1219 \\
584\end{array}$ & $59-61$ \\
\hline Virginia & Ultisols (sil) & $\mathrm{C}_{\mathrm{s}}-\mathrm{C}_{\mathrm{s}}$ & 1966-1971 & $0-15$ & $\begin{array}{l}224 \\
224\end{array}$ & $\begin{array}{r}85 \\
124\end{array}$ & $\begin{array}{l}-139 \\
-100\end{array}$ & $\begin{array}{r}0 \\
336\end{array}$ & $\begin{array}{l}411 \\
440\end{array}$ & $\begin{array}{l}-411 \\
-104\end{array}$ & 62 \\
\hline \multirow[t]{2}{*}{ China } & Inceptisols & $\mathrm{W}_{\mathrm{w}}-\mathrm{C}^{4}$ & $1990-2005$ & $0-20$ & $\begin{array}{l}28(4) \\
30(3)\end{array}$ & $\begin{array}{r}5(3) \\
24(8)\end{array}$ & $\begin{array}{r}-23(1) \\
-5(5)\end{array}$ & $\begin{array}{r}0 \\
1500\end{array}$ & $\begin{array}{r}718 \\
1089\end{array}$ & $\begin{array}{r}-718 \\
411\end{array}$ & 63,64 \\
\hline & Inceptisols (sl) & $\mathrm{C}-\mathrm{C}$ & 1993-2004 & $0-20$ & $\begin{array}{l}206 \\
206\end{array}$ & $\begin{array}{l}223 \\
235\end{array}$ & $\begin{array}{l}17 \\
29\end{array}$ & $\begin{array}{r}0 \\
259\end{array}$ & $\begin{array}{l}480 \\
650\end{array}$ & $\begin{array}{l}-480 \\
-391\end{array}$ & 65,66 \\
\hline \multirow[t]{3}{*}{ England } & Alfisols (sicl) & $\mathrm{B}-\mathrm{B}^{5}$ & $1856-1974$ & $0-23$ & $\begin{array}{l}313 \\
313\end{array}$ & $\begin{array}{l}219 \\
305\end{array}$ & $\begin{array}{r}-94 \\
-8\end{array}$ & $\begin{array}{r}0 \\
5040\end{array}$ & $\begin{array}{l}1221 \\
4294\end{array}$ & $\begin{array}{r}-1221 \\
746\end{array}$ & 67 \\
\hline & Inceptisols (sl) & $\mathrm{Sb}-\mathrm{W}_{\mathrm{w}}-\mathrm{B}$ & 1965-1982 & $0-25$ & $\begin{array}{l}162 \\
162\end{array}$ & $\begin{array}{l}132 \\
265\end{array}$ & $\begin{array}{r}-30 \\
102\end{array}$ & $\begin{array}{r}0 \\
1992\end{array}$ & $\begin{array}{r}580 \\
1160\end{array}$ & $\begin{array}{r}-580 \\
832\end{array}$ & 68 \\
\hline & Alfisols (sicl) & $\mathrm{W}_{\mathrm{w}}-\mathrm{W}_{\mathrm{w}}$ & 1966-1992 & $0-23$ & $\begin{array}{r}323 \\
1326\end{array}$ & $\begin{array}{r}271 \\
1236\end{array}$ & $\begin{array}{l}-52 \\
-90\end{array}$ & $\begin{array}{r}0 \\
2430\end{array}$ & $\begin{array}{r}675 \\
1431\end{array}$ & $\begin{array}{r}-675 \\
999\end{array}$ & 69 \\
\hline
\end{tabular}




\begin{tabular}{|c|c|c|c|c|c|c|c|c|c|c|c|}
\hline \multirow[b]{2}{*}{ Location } & \multirow[b]{2}{*}{ Soil order ${ }^{1}$} & \multirow{2}{*}{$\begin{array}{l}\text { Cropping } \\
\text { system }^{2}\end{array}$} & \multirow{2}{*}{$\begin{array}{l}\text { Study } \\
\text { period }\end{array}$} & \multirow{2}{*}{$\begin{array}{l}\text { Sampling } \\
\text { depth }\end{array}$} & \multicolumn{3}{|c|}{ Exchangeable $\mathrm{K}^{3}$} & \multicolumn{3}{|c|}{ Cumulative } & \multirow[b]{2}{*}{ Reference(s) } \\
\hline & & & & & Initial & Final & Net change & $\mathrm{K}$ added & $\mathrm{K}$ removed & Net $K$ input & \\
\hline \multirow[t]{4}{*}{ Denmark } & Alfisols (s) & $\mathrm{W}_{\mathrm{w}}-\mathrm{Rcl}-\mathrm{B}-\mathrm{Gcl}$ & 1949-1972 & $0-20$ & 65 & 52 & -13 & 0 & 456 & -456 & 70 \\
\hline & & & & & 188 & 172 & -15 & 1584 & 1584 & 0 & \\
\hline & Alfisols (sl) & $\mathrm{W}_{\mathrm{w}}-\mathrm{Rcl}-\mathrm{B}-\mathrm{Gcl}$ & 1949-1972 & $0-20$ & 98 & 62 & -36 & 0 & 696 & -696 & \\
\hline & & & & & 152 & 122 & -30 & 1584 & 2160 & -576 & \\
\hline \multirow[t]{4}{*}{ Germany } & Alfisols (sil) & $\mathrm{Rc}-\mathrm{Ce}-\mathrm{Ce}$ & 1914-1975 & $0-15$ & 158 & 123 & -35 & 0 & 4531 & -4531 & 71 \\
\hline & & & & & 158 & 131 & -27 & 5127 & 7039 & -1912 & \\
\hline & Mollisols (sl) & $\mathrm{Po}-\mathrm{P}-\mathrm{W}_{\mathrm{s}}-\mathrm{Rc}-\mathrm{B}$ & 1977-1984 & $0-20$ & 170 & 179 & 9 & 0 & 568 & -568 & 72 \\
\hline & & & & & 496 & 448 & -48 & 1792 & 1256 & 536 & \\
\hline \multirow[t]{4}{*}{ India } & Inceptisols (ls) & $\mathrm{C}-\mathrm{W}_{\mathrm{w}}-\mathrm{Cp}^{4}$ & 1971-1996 & $0-15^{6}$ & $8(8)$ & $7(0)$ & $-1(8)$ & 0 & 3952 & -3952 & 73 \\
\hline & & & & & $9(6)$ & $9(8)$ & (2) & 2860 & 5642 & -2782 & \\
\hline & Alfisols & $\mathrm{C}-\mathrm{W}_{\mathrm{w}}^{4}$ & 1972-1996 & $0-15$ & 194 & 148 & -46 & 0 & 1508 & -1508 & 74 \\
\hline & & & & & 194 & 167 & -27 & 2460 & 2393 & 67 & \\
\hline New Zealand & Inceptisols & $\mathrm{A}-\mathrm{A}^{4}$ & 1971-1974 & $0-20$ & 216 & 225 & 9 & 0 & 571 & -571 & 75 \\
\hline & & & & & 216 & 394 & 178 & 786 & 758 & 28 & \\
\hline Nigeria & Alfisols $\left(\mathrm{sc}_{1}, \mathrm{ls}_{2}\right)$ & $C-C^{4}(3)$ & 1966-1968 & $0-15$ & 381 & 198 & -183 & 0 & 197 & -197 & 76 \\
\hline & & & & & 381 & 228 & -153 & 168 & 239 & -71 & \\
\hline \multirow[t]{6}{*}{ Philippines } & Inceptisols (sic) & $\mathrm{Ri}-\mathrm{Ri}$ & 1968-1986 & $0-20$ & 521 & $13(5)$ & $-38(6)$ & 0 & $578^{7}$ & -578 & 77 \\
\hline & & & & & 521 & $15(6)$ & $-36(5)$ & 950 & $759^{7}$ & 191 & \\
\hline & Vertisols (c) & $\mathrm{Ri}-\mathrm{Ri}$ & 1968-1986 & $0-20$ & 209 & $24(9)$ & $4(0)$ & 0 & $580^{7}$ & -580 & \\
\hline & & & & & 209 & $39(3)$ & $18(4)$ & 950 & $799^{7}$ & 151 & \\
\hline & Vertisols (c) & $\mathrm{Ri}-\mathrm{Ri}$ & 1968-1986 & $0-20$ & 938 & $57(3)$ & $-36(5)$ & 0 & $531^{7}$ & -531 & \\
\hline & & & & & 938 & $62(6)$ & $-31(2)$ & 950 & $677^{7}$ & 273 & \\
\hline \multirow[t]{4}{*}{ Poland } & Alfisols (ls) & Ry-Ry & 1963-1995 & $0-25$ & 107 & 200 & 93 & 0 & 1254 & -1254 & 69 \\
\hline & & & & & 320 & 300 & -20 & 2574 & 2310 & 264 & \\
\hline & & Po-B-Rcl-W ${ }_{\mathrm{w}}-\mathrm{Ry}$ & 1963-1995 & $0-25$ & 113 & 147 & 33 & 0 & 1584 & -1584 & \\
\hline & & & & & 327 & 363 & 37 & 2574 & 3069 & -495 & \\
\hline \multirow[t]{2}{*}{ Scotland } & Spodosols (ls) & $\operatorname{Pr}-\operatorname{Pr}^{8}$ & 1969-1998 & $0-15$ & 72 & 20 & -52 & $448^{9}$ & 1608 & -1160 & 78 \\
\hline & & & & & 55 & 24 & -31 & $1969^{9}$ & 3158 & -1189 & \\
\hline
\end{tabular}

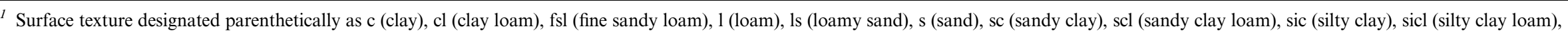
sil (silt loam) and sl (sandy loam). Subscripts indicate the number of soils studied with a given texture.

${ }_{2}$ A, alfalfa (Medicago sativa L.); B, barley (Hordeum vulgare L.); C, corn (Zea mays L.); Cb, coastal bermudagrass (Cynodon dactylon L. Pers.); Ce, cereal; Cp, cowpea (Vigna unguiculata L.); $\mathrm{C}_{\mathrm{s}}$, silage corn (Zea mays L.); Ct, cotton (Gossypium hirsutum L.); Gcl, grass-clover; L, ley; P, pea (Pisum L.); Pm, pearl millet (Pennisetum glaucum L. R. Br.); Po, potato (Solanum tuberosum L.); Pr, perennial ryegrass (Lolium perenne L.); Rc, root crop; Rcl, red clover (Trifolium pratense L.); Ri, rice (Oryza sativa L.); Ry, rye (Secale cereale L.); S, soybean (Glycine max L. Merr.); Sb, sugarbeet (Beta vulgaris $\mathrm{L}$.); Sp, sweet potato (Ipomoea batatas); $\mathrm{W}_{\mathrm{s}}$, spring wheat (Triticum aestivum $\mathrm{L}$.); $\mathrm{W}_{\mathrm{w}}$, winter wheat (Triticum aestivum $\mathrm{L}$.). Values in parentheses indicate the number of separate trials summarized.

${ }_{3}^{3}$ Parentheses indicate digital uncertainty in estimating data reported in figures.

4 All crops were grown annually.

Prior to 1901, the cropping system was $\mathrm{W}_{\mathrm{w}}-\mathrm{W}_{\mathrm{w}}(1856-1875)$ or Po-Po (1876-1901).

${ }^{6}$ Assumed as the depth for sampling surface soil.

${ }^{7}$ Rice grain was assumed to contain $0.04 \mathrm{~g} \mathrm{~K} \mathrm{~kg}^{-1}$ at a moisture content of $140 \mathrm{~g} \mathrm{~kg}^{-1} 79$.

${ }^{8}$ Cropped to cereals between 1982 and 1987, with 4 years of barley, 1 year of oats (Avena sativa L.) and 1 year of winter wheat.

9 Includes atmospheric K deposition. 
Table 3. Speciation of potassium in surface $(0-18 \mathrm{~cm})$ samples collected in August and September between 1986 and 1989 from a Drummer soil under a corn-soybean rotation with no P or K fertilization since $1970 .{ }^{1}$

\begin{tabular}{|c|c|c|c|c|c|c|c|c|c|}
\hline \multirow[b]{2}{*}{ Year } & \multirow[b]{2}{*}{ 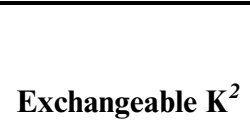 } & \multicolumn{7}{|c|}{ Non-exchangeable $\mathrm{K}$ recovered in six successive extractions ${ }^{3}$} & \multirow[b]{2}{*}{ Total $\mathbf{K}^{4}$} \\
\hline & & Ext. 1 & Ext. 2 & Ext. 3 & Ext. 4 & Ext. 5 & Ext. 6 & Ext. 1-6 & \\
\hline & & & & & & $a^{-1}$ & & & \\
\hline 1986 & $240(16)$ & 1048 (54) & $616(58)$ & $463(37)$ & $351(8)$ & $252(37)$ & $239(28)$ & 2969 & $42,271(682)$ \\
\hline 1987 & 244 (18) & $932(47)$ & $588(43)$ & $407(35)$ & $435(16)$ & $351(16)$ & $308(24)$ & 3021 & ND \\
\hline 1988 & $224(23)$ & $1120(50)$ & $700(56)$ & $476(8)$ & $336(32)$ & $379(21)$ & $267(8)$ & 3278 & ND \\
\hline 1989 & $276(25)$ & 987 (24) & $627(35)$ & 448 (16) & $358(14)$ & 299 (29) & $269(16)$ & 2988 & $42,208(680)$ \\
\hline
\end{tabular}

$1160-180 \mathrm{~kg} \mathrm{Nha}^{-1}$ applied for corn production in 1986 and 1988. All values reported as a mean of triplicate determinations performed on a sample composited from 45 to 75 cores collected in August and September of each year. Standard deviations shown in parentheses.

${ }_{2}^{2}$ Determined by extraction with $1 \mathrm{M} \mathrm{NH}_{4} \mathrm{C}_{2} \mathrm{H}_{3} \mathrm{O}_{2}(\mathrm{pH} 7)^{24}$ after air drying at $40^{\circ} \mathrm{C}$.

3 Determined after extraction of exchangeable $\mathrm{K}$, by boiling with $1 \mathrm{M} \mathrm{HNO}_{3}{ }^{24}$.

${ }^{4}$ Determined by fusion with $\mathrm{LiBO}_{2} \cdot 8 \mathrm{H}_{2} \mathrm{O}$, followed by dissolution in $1 \mathrm{M} \mathrm{HNO}_{3}{ }^{25}$. ND, not determined.

\section{Fertilizer value of potassium chloride}

Besides being abundant in soils and plant residues, $\mathrm{K}$ is notable as the only macronutrient dominated by inorganic forms in both the soil and plant, and thus availability is not dependent upon microbial transformations. Uptake occurs much more readily for $\mathrm{K}^{+}$than for $\mathrm{Ca}^{2+}$ or $\mathrm{Mg}^{2+}$ because of greater membrane permeability further enhanced at low concentrations by active diffusion, while specialized transport systems selective for $\mathrm{K}$ rapidly distribute this nutrient in the plant ${ }^{33,107}$. Under these circumstances, and considering the ubiquitous nature of $\mathrm{K}$, it becomes clear why many years can be required to induce fertilizer $\mathrm{K}$ response in long-term static plot experiments ${ }^{59,60,71,77,108}$ (see also supplemental references [46-52] for the online version of the paper). On the basis of such findings, there is little reason to expect economically viable crop response to shorter periods of $\mathrm{K}$ fertilization that limit check-plot depletion, and a far lower likelihood of response in a production setting where the entire field shares the same history of regular fertilizer $\mathrm{K}$ inputs.

This view was indeed confirmed by compiling a global database of crop response to $\mathrm{KCl}$ from 2121 short-term field trials conducted by public universities or experiment stations. The resulting database is too extensive to be accommodated herein but is available in the online supplement as Table S4 (for the online version of the paper). As hypothesized, $\mathrm{KCl}$ fertilization was often ineffective for increasing productivity, according to nonsignificant responses that occurred in approximately $76 \%$ of the total trials surveyed. The inherent capability for plant uptake of soil $\mathrm{K}$ is manifestly evident from studies in which shallow-rooted crops growing on sandy soils were non-responsive to $\mathrm{K}$ fertilization ${ }^{109-112}$, and often made fertilizer $\mathrm{K}$ superfluous when deeper rooted crops had access to profile $\mathrm{K}$ supplies in more productive soils. Unfortunately, $\mathrm{KCl}$ usage in the latter case has long been exacerbated by a buildup-maintenance philosophy that promotes intensive fertilizer $\mathrm{K}$ inputs without regard to huge soil $\mathrm{K}$ reserves or their recycling through crop residues.

To ascertain the value of $\mathrm{K}$ fertilization, those trials showing a statistically significant response to $\mathrm{KCl}$ fertilization, accounting for $24 \%$ of the total database, are presented herein in Table 4. Most of the responses were positive and occurred on coarse-textured, organic or highly weathered soils inherently low in K-supplying power (231 site-years); when the above-ground residues were removed (191 site-years); with crops having a shallow or low-density rooting system (62 site-years); and/or when subsoil rooting was restricted (12 site-years). In the absence of such factors, there is very little reason to expect a significant yield response to $\mathrm{KCl}$ fertilization.

The input-intensive approach to fertilizer $\mathrm{K}$ management can have negative economic consequences for producers, which are apt to go unnoticed unless fertilizer response is determined relative to yield in the absence of fertilization. These consequences are to be expected considering the ample evidence in Table S4 (for the online version of the paper) that $\mathrm{KCl}$ inputs are often ineffective for increasing yield, but will be more serious when yield is depressed. The latter effect has indeed been observed, and was significant in field studies with $\operatorname{corn}^{38,144,148,192}$, soybean $^{120,125,131,175}$, wheat (Triticum aestivum L.) ${ }^{186}$, sugarbeet (Beta vulgaris L.) ${ }^{139}$, sugarcane (Saccharum officinarum L.) ${ }^{127}$, alfalfa ${ }^{145}$, peanut (Arachis hypogaea L.) ${ }^{109,191}$, rape (Brassica napus L. $)^{176}$ and cowpea (Vigna unguiculata L.) ${ }^{166}$. In several of these studies, the loss of yield was intensified by increasing the rate of $\mathrm{KCl}$ application $^{38,109,120,125,175,192}$, and in some cases the higher rate transformed significant yield gain to loss ${ }^{127,186}$.

Yield reductions due to $\mathrm{KCl}$ fertilization, as documented in Table 4, can be explained by the high salt index of this fertilizer, which has been implicated as a detrimental factor for crop germination and growth ${ }^{193-195}$ and microbial processes ${ }^{196,197}$. More serious consequences can occur because of the anion supplied by $\mathrm{KCl}$. 


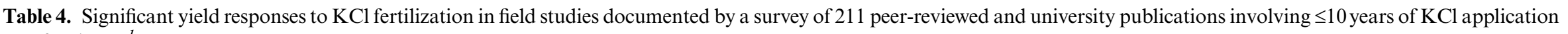
at a fixed rate. ${ }^{l}$

\begin{tabular}{|c|c|c|c|c|c|c|c|c|c|}
\hline \multirow[b]{2}{*}{ Location } & \multirow[b]{2}{*}{ Soil order ${ }^{2}$} & \multirow{2}{*}{$\begin{array}{l}\text { Exchangeable } \\
\mathrm{K}^{3}\end{array}$} & \multirow{2}{*}{$\begin{array}{l}\text { K applied } \\
\text { (as KCl) }\end{array}$} & \multirow{2}{*}{$\begin{array}{l}\text { Site- } \\
\text { years } \\
\text { studied }^{4}\end{array}$} & \multirow{2}{*}{$\begin{array}{l}\text { Cropping } \\
\text { system }^{5}\end{array}$} & \multirow{2}{*}{$\begin{array}{l}\text { Yield } \\
\text { without } \\
\text { fertilizer } \mathrm{K}^{6}\end{array}$} & \multicolumn{2}{|c|}{ Yield response to $K^{7}$} & \multirow[b]{2}{*}{ Reference } \\
\hline & & & & & & & Lowest & Highest & \\
\hline & & $\mathrm{kgha}^{-1}$ & $\mathrm{kgha}^{-1} \mathrm{yr}^{-1}$ & & & $\mathrm{Mgha}^{-1}$ & - & $\%$ & \\
\hline \multicolumn{10}{|c|}{ North America } \\
\hline Manitoba & Mollisols (scl) & $50-310(15)$ & $0-200$ & 15 & $\mathrm{~A}^{9}$ & 16.7 & $59 *(50)$ & $174 *(200)$ & 113 \\
\hline Ontario & Alfisols (sl) & NR & $0-126$ & 4 & $\overline{\mathrm{O}}-\underline{\mathrm{Po}}$ & 19.8 & $24 *(126)$ & $24 *(126)$ & 114 \\
\hline \multicolumn{10}{|l|}{ USA } \\
\hline \multirow[t]{2}{*}{ Alabama } & Ultisols (sil) & 42 & $0-150$ & 3 & $\mathrm{Bg}^{9}-\underline{\mathrm{S}}$ & 2.6 & $27 *(75)$ & $44 *(150)$ & 115 \\
\hline & Ultisols (fsl, sil) & $25-42^{8}(20)$ & $0-112$ & 6 & $\mathrm{Tf}, \mathrm{W}^{9}{ }^{9}$ & 3.6 & $25^{\mathrm{ns}}(28)$ & $46^{*}(112)$ & 116 \\
\hline Alaska & Inceptisols (sil) & 256 & $0-186$ & 2 & Sbg ${ }^{9}$ & 3.2 & $-15^{\text {ns }}(112)$ & $31 *(186)$ & 117 \\
\hline \multirow[t]{3}{*}{ Arkansas } & Alfisols (sil) & $112(16)$ & $0-75$ & 3 & $\underline{\mathrm{S}}$ & 2.8 & $8 *(75)$ & $8 *(75)$ & 118 \\
\hline & Alfisols (fsl) & $213-269(15)$ & $0-84$ & 6 & $\overline{\mathrm{C}} \mathrm{t}$ & 2.1 & $10 *(28)$ & $22 *(84)$ & 119 \\
\hline & Alfisols (sil) & NR & $0-1175$ & 8 & $\underline{\bar{S}}$ & 2.7 & $-21 *(1175)$ & $-9^{\text {ns }}(587)$ & 120 \\
\hline \multirow[t]{2}{*}{ California } & Mollisols (sl) & $65^{8}$ & $0-480$ & 3 & $\mathrm{Ct}$ & 2.(4) & $11^{* *}(120)$ & $31 * * *(480)$ & 121 \\
\hline & Alfisols (1) & $67^{8}(20)$ & $0-100$ & 3 & $\overline{\mathrm{Ri}}^{9}$ & 7.(8) & $5^{*}(63)$ & $7 *(100)$ & 122 \\
\hline Colorado & Mollisols (cl) & $335^{8}(15)$ & $0-750$ & 16 & $\overline{\mathrm{A}^{9}}$ & 52.7 & $6^{*}(375,750)$ & $6^{*}(375,750)$ & 123 \\
\hline Connecticut & Inceptisols (fsl) & $121^{7}$ & $0-448$ & 6 & $\overline{\mathrm{R}} \mathrm{c}^{9}$ & 10.7 & $7 *(448)$ & $12 *(224)$ & 124 \\
\hline Delaware & Ultisols (sil) & 207 & $0-223$ & 20 & $\bar{S}$ & 2.0 & $-30 *(223)$ & $-11 *(56)$ & 125 \\
\hline \multirow[t]{3}{*}{ Florida } & Spodosols (fs) & $45-52^{8}(15)$ & $0-200$ & 1 & $\overline{\mathrm{S}}-\mathrm{Vg}$ & 4.0 & $6^{\text {ns }}(50)$ & $15 *(200)$ & 126 \\
\hline & Histosols & $43(30)$ & $0-560$ & 1 & $\overline{\mathrm{Sc}}^{9}$ & 97.2 & $-4 * *(560)$ & $6^{* *}(280)$ & 127 \\
\hline & Ultisols (lfs) & $17-150^{8}(15)$ & $0-418$ & 2 & $\underline{\mathrm{C}}-\left(\mathrm{S}\right.$ or $\left.\mathrm{Ar}^{9}\right)$ & 9.5 & $19 *(209)$ & $21 *(418)$ & 128 \\
\hline \multirow[t]{5}{*}{ Georgia } & Ultisols (sl) & NR & $0-188$ & 3 & $\overline{\mathrm{Bg}}^{9}$ & 9.5 & $11 *(47)$ & $14 *(188)$ & 129 \\
\hline & Ultisols (fs) & $30(15)$ & $0-168$ & 6 & $\mathrm{Pn}$ & 2.3 & $17 *(34)$ & $34 *(168)$ & 130 \\
\hline & Ultisols (ls, sl) & $41-182$ & $0-224$ & 9 & $\mathrm{Pn}$ & 4.(2) & $-4 *(224)$ & $-1^{\mathrm{ns}}(121)$ & 109 \\
\hline & Ultisols (s) & NR & $0-186$ & 30 & $\mathrm{~S}$ & 1.7 & $-30 *(186)$ & $-30 *(186)$ & 131 \\
\hline & Entisols (ls) & $10^{8}(25)$ & $0-56$ & 3 & $\overline{\mathrm{C}}-\underline{\mathrm{Pn}}$ & 3.5 & $47 *(56)$ & $47 *(56)$ & 132 \\
\hline Idaho & Mollisols (gl) & $92-112^{8}(20)$ & $0-415$ & 5 & $\mathrm{~A}^{9}-\underline{\mathrm{Po}}$ & 24.8 & $20 *(415)$ & $21 *(208)$ & 133 \\
\hline \multirow[t]{3}{*}{ Illinois } & Alfisols (sil) & $60-86(15)$ & $0-224$ & 2 & $\mathrm{Sg}-\overline{\mathrm{C}}$ & 4.1 & $2^{\mathrm{ns}}(28)$ & $60 * *(224)$ & 38 \\
\hline & Alfisols (sil) & 63-161 (15) & $0-224$ & 2 & $\mathrm{C}$ & 4.9 & $20 * *(28)$ & $118 * *(112)$ & 134 \\
\hline & Inceptisols (sil) & $166(15)$ & $0-112$ & 1 & $\overline{\mathrm{C}}$ & 8.3 & $-1^{\mathrm{ns}}(28)$ & $7 *(56)$ & \\
\hline \multirow[t]{2}{*}{ Indiana } & Alfisols (fs) & $51^{8}(18)$ & $0-372$ & 3 & $\mathrm{~A}^{9}$ & 3.7 & $32 * *(93)$ & $54 * *(372)$ & 135 \\
\hline & Alfisols (sil) & $74^{8}(18)$ & $0-372$ & 2 & $\underline{\mathrm{A}}^{9}$ & 5.1 & $15 *(93)$ & $21 *(372)$ & \\
\hline \multirow[t]{2}{*}{ Iowa } & Mollisols (sl-sil) & $141-491(15)$ & $0-335$ & 9 & $\mathrm{~A}^{9}$ & 4.3 & $1^{\mathrm{ns}}(74,112)$ & $12 *(335)$ & 136 \\
\hline & Alfisols (sil) & $62-190(15)$ & $0-140$ & 5 & $\mathrm{~A}^{9}-\mathrm{C}$ & 5.7 & $10 *(28)$ & $16^{*}(112)$ & 38 \\
\hline Kansas & Alfisols (sil) & $45-108^{8}(15)$ & $0-140$ & 6 & $\left(\mathrm{~A}^{9}\right.$ or $\left.\mathrm{Bt}^{9}\right)-\mathrm{Sr}$ & $6 .(0)$ & $10 *(70,140)$ & $10 *(70,140)$ & 137 \\
\hline Kentucky & Ultisols (sil) & NR & $0-372$ & 3 & $\mathrm{Tf} / \mathrm{Wc}^{9}$ & 5.1 & $0^{\text {ns }}(372)$ & $7 *(140)$ & 138 \\
\hline \multirow[t]{2}{*}{ Michigan } & Alfisols (sl, l) & $77-242(15)$ & $0-335$ & 7 & $\underline{\mathrm{A}}, \underline{\mathrm{Rcl}}, \mathrm{Sbg}^{9}$ & 4.1 & $8^{\text {ns }}(19)$ & $33^{*}(335)$ & 136 \\
\hline & Alfisols (1) & NR & $93-186$ & 1 & $\underline{\mathrm{Sb}}$ & $50.7^{10}$ & $-6^{*}(186)$ & $-6^{*}(186)$ & 139 \\
\hline \multirow[t]{4}{*}{ Minnesota } & Alfisols (sil) & $102-142(15)$ & $0-140$ & 2 & $\overline{A^{9}}-\underline{C}$ & 5.2 & $10^{\mathrm{ns}}(28)$ & $25 *(140)$ & 38 \\
\hline & Entisols (fs) & $122(15)$ & $0-140$ & 1 & $\mathrm{~A}^{9}-\overline{\mathrm{C}}$ & 3.9 & $-18 *(140)$ & $3^{\text {ns }}(28)$ & \\
\hline & Histosols & $37(15)$ & $0-140$ & 1 & $\mathrm{C}-\overline{\mathrm{C}}$ & 2.4 & $38 *(140)$ & $46^{*}(84,112)$ & \\
\hline & Mollisols (ls, sil) & $111-198(15)$ & $0-140$ & 2 & $\mathrm{~A}^{9-}-\underline{C}$ & 4.6 & $16^{*}(28)$ & $37 * *(140)$ & \\
\hline
\end{tabular}




\begin{tabular}{|c|c|c|c|c|c|c|c|c|}
\hline \multirow[t]{4}{*}{ Mississippi } & Entisols (sil) & NR & $0-112$ & 2 & $\mathrm{C}-\mathrm{Ct}$ & 1.3 & $17 *(37)$ & $31 *(112)$ \\
\hline & Inceptisols (fsl) & $289-465(15)$ & $0-112$ & 8 & $\mathrm{Ct}^{-}$ & 1.0 & $9 *(112)$ & $9 *(112)$ \\
\hline & Entisols (si) & $115^{8}(15)$ & $0-204$ & 2 & $\overrightarrow{\mathrm{Ct}}$ & 1.2 & $7 *(68)$ & $13 *(204)$ \\
\hline & Inceptisols (fsl) & $138-159^{8}(15)$ & $0-112$ & 11 & $\underline{\mathrm{Ct}}$ & 1.1 & $5^{*}(112)$ & $5 *(112)$ \\
\hline Nebraska & Entisols $_{1}$, Mollisols $_{15}$ & $91-609^{8}(20)$ & $0-40$ & 16 & $\underline{\mathrm{C}}-\mathrm{S}$ & 14.4 & $-3^{*}(40)$ & $-3^{*}(40)$ \\
\hline New Mexico & Entisols (fsl) & NR & $0-56$ & 3 & $\overline{\mathrm{A}}^{9}$ & 16.6 & $-2 *(56)$ & $-2 *(56)$ \\
\hline North Carolina & Ultisols (fsl) & $12^{8}$ & $0-112$ & 3 & $\overline{\mathrm{C}}-\mathrm{S}$ & 0.35 & $131 *(11)$ & $342 *(112)$ \\
\hline North Dakota & Mollisols (1) & $570-940$ & 0-99 & 4 & $\underline{\mathrm{B}}$ & 2.7 & $1^{\mathrm{ns}}(24)$ & $4 *(99)$ \\
\hline \multirow[t]{2}{*}{ Ohio } & Mollisols (sicl) & NR & $0-360$ & 2 & C & 9.2 & $-20 * * *(90)$ & $-19 * * *(360)$ \\
\hline & Alfisols (sil) & 190 & $0-74$ & 6 & $\overline{\mathrm{C}}-\underline{\mathrm{S}}$ & 3.0 & $1^{\mathrm{ns}}(37)$ & $7 *(74)$ \\
\hline \multirow[t]{2}{*}{ Pennsylvania } & Alfisols (sicl) & $32(15)$ & $0-112$ & 6 & $\mathrm{Og}^{\overline{9}}$ & 4.0 & $21 *(56)$ & $29 *(112)$ \\
\hline & $\begin{array}{l}\text { Alfisols }_{4} \text { (sil), } \\
\text { Ultisols }_{2} \text { (sil) }\end{array}$ & $61-140(15)$ & $0-525$ & 16 & ${\underline{\mathrm{A}^{9}}}^{9}$ & 2.(8) & $66 *(175)$ & $72 *(350)$ \\
\hline South Dakota & Mollisols & $>391$ & $0-71$ & 20 & $\underline{\mathrm{O}}$ & 2.5 & $3 *(35,71)$ & $3 *(35,71)$ \\
\hline \multirow[t]{2}{*}{ Tennessee } & Mollisols (fsl) & NR & $0-186$ & 1 & $\overline{\mathrm{C}}$ & 5.0 & $-2^{\text {ns }}(149)$ & $18 *(19)$ \\
\hline & Alfisols (sil) & $198-444(15)$ & $56-112$ & 6 & $\overline{\mathrm{C}} \mathrm{t}$ & $1.8^{10}$ & $5 *(112)$ & $5 *(112)$ \\
\hline Texas & Ultisols (fs, fsl) & NR & $0-278$ & 6 & $\overline{\mathrm{Bg}}^{9}$ & 10.3 & $24 *(139,278)$ & $24 *(139,278)$ \\
\hline Utah & Mollisols (sil) & $59-77^{8}(30)$ & $0-224$ & 2 & Po & 37.4 & $15^{*}(224)$ & $15 *(224)$ \\
\hline \multirow[t]{2}{*}{ Virginia } & Ultisols (sl) & $55(15)$ & $0-372$ & 6 & $\overline{\mathrm{S}}$ & 1.8 & $25 *(372)$ & $25 *(372)$ \\
\hline & Ultisols $(\mathrm{cl})$ & $66-121$ & $0-112$ & 2 & $\overline{\mathrm{S}}$ & 1.6 & $85 *(28)$ & $139 *(112)$ \\
\hline \multirow[t]{5}{*}{ Wisconsin } & Mollisols (sil) & $142(15)$ & $0-1120$ & 3 & $\bar{A}^{9}$ & 6.5 & $22 *(56)$ & $53 *(672)$ \\
\hline & Alfisols (sil) & $124(15)$ & $0-1792$ & 2 & $\overline{\mathrm{A}}^{9}$ & 8.3 & $11^{\mathrm{ns}}(1344)$ & $32 *(448)$ \\
\hline & Mollisols (sil) & $167(15)$ & $0-896$ & 1 & $\mathrm{~A}^{9}$ & 6.2 & $14 *(896)$ & $22 *(448)$ \\
\hline & Mollisols (sil) & $142(15)$ & $0-672$ & 2 & $\underline{A}^{9}$ & 5.8 & $34 *(224)$ & $37 *(672)$ \\
\hline & Entisols (ls) & $72-90^{8}$ & $0-372$ & 4 & $\underline{\mathrm{Po}}$ & 34.8 & $17 *(93)$ & $25 *(280)$ \\
\hline \multicolumn{9}{|l|}{ South America } \\
\hline \multirow[t]{2}{*}{ Brazil } & Oxisols & $46^{8}$ & $0-168$ & 9 & $\mathrm{Ri}-\mathrm{Ri}$ & 1.1 & $9 *(42)$ & $19 * *(126)$ \\
\hline & Oxisols (1s) & $20-52^{8}(20)$ & $0-199$ & 2 & $\mathrm{~S}$ & 1.(5) & $65 *(33)$ & $111 *(133)$ \\
\hline \multirow[t]{2}{*}{ Peru } & Ultisols & $55^{8}(15)$ & $0-120$ & 6 & $\overline{\mathrm{R}} \mathrm{i}-\mathrm{Cp}^{9}$ & 2.1 & $4^{\mathrm{ns}}(20)$ & $22 *(80)$ \\
\hline & & $55^{8}(15)$ & $0-120$ & 4 & $\mathrm{Ri}-\mathrm{Cp}^{9}$ & 0.8 & $-24 *(20)$ & $25 *(120)$ \\
\hline Venezuela & Oxisols (scl) & $38^{8}$ & $0-135$ & 2 & $\underline{S}$ & 1.0 & $8^{\text {ns }}(9)$ & $173 *(108)$ \\
\hline \multicolumn{9}{|l|}{ Europe } \\
\hline Macedonia & Entisols & $83^{8}(20)$ & $0-100$ & 2 & $\underline{\text { Po }}$ & 33.3 & $13^{* *}(100)$ & $13^{* *}(100)$ \\
\hline \multicolumn{9}{|l|}{ Africa } \\
\hline Madagascar & Oxisols (sl) & $55^{8}(20)$ & $0-75$ & 5 & $\mathrm{~S}$ & 0.5 & $278 * *(25)$ & $422 * *(50,75)$ \\
\hline \multirow[t]{3}{*}{ Nigeria } & Oxisols (ls-sl) & $31-817^{8}(15)$ & $0-75$ & 9 & $\overline{\mathrm{C}} \mathrm{t}$ & 1.9 & $35 *(25)$ & $42 *(75)$ \\
\hline & Alfisols, Ultisols (ls) & $39-78^{8}(15)$ & $0-40$ & 20 & $\overline{\mathrm{Gn}}$ & 1.4 & $24 *(20)$ & $31 *(40)$ \\
\hline & Inceptisols (ls) & $20-66^{8}(15)$ & $0-40$ & 20 & $\mathrm{Gn}$ & 1.1 & $20 *(20)$ & $22 *(40)$ \\
\hline \multicolumn{9}{|l|}{ Asia } \\
\hline \multirow[t]{3}{*}{ Bangladesh } & Inceptisols (scl) & $20^{8}$ & $28-37$ & 1 & $\mathrm{Sa}$ & $1.3^{10}$ & $8 *(37)$ & $8 *(37)$ \\
\hline & Entisols (scl) & $78-86^{8}(30)$ & $0-180$ & 2 & $\overline{\mathrm{Sc}^{9}}$ & 74.8 & $6 *(45,135)$ & $9 *(70,90)$ \\
\hline & Inceptisols (sicl) & $133^{8}(20)$ & $66-133$ & 1 & $\underline{\mathrm{Ra}}^{9}$ & $24.0^{10}$ & $8 *(100)$ & $12 *(133)$ \\
\hline \multirow[t]{6}{*}{ India } & Vertisols & NR & $9-150$ & 2 & $\underline{\mathrm{S}}$ & $3.1^{10}$ & $-7 *(37)$ & $-3^{*}(19)$ \\
\hline & Inceptisols (sl) & 223 & $0-33$ & 1 & $\overline{\mathrm{R}}^{9}$ & 1.1 & $-20 *(33)$ & $-20 *(33)$ \\
\hline & Alfisols, Inceptisols & $22-60^{8}(15)$ & $0-156$ & 6 & $\overline{\mathrm{W}}_{\mathrm{w}}{ }^{9}$ & 4.(8) & $3^{\mathrm{ns}}(156)$ & $6 * *(104)$ \\
\hline & Inceptisols (s-sl) & $56-158^{8}$ & $0-84$ & 28 & $\underline{\mathrm{Po}}_{9}^{\mathrm{W}}$ & 12.1 & $15^{*}(42)$ & $22 *(84)$ \\
\hline & Inceptisols (ls, sil) & NR & $0-66$ & 4 & $\overline{\mathrm{Ri}}^{9}$ & 5.3 & $6^{*}(33)$ & $11 *(66)$ \\
\hline & & & & 4 & $\overline{\mathrm{W}}_{\mathrm{w}}{ }^{9}$ & 3.0 & $12 *(33)$ & $24 *(66)$ \\
\hline
\end{tabular}




\begin{tabular}{|c|c|c|c|c|c|c|c|c|c|}
\hline \multirow[b]{2}{*}{ Location } & \multirow[b]{2}{*}{ Soil order ${ }^{2}$} & \multirow{2}{*}{$\begin{array}{l}\text { Exchangeable } \\
\mathbf{K}^{3}\end{array}$} & \multirow{2}{*}{$\begin{array}{l}\mathrm{K} \text { applied } \\
\text { (as KCl) }\end{array}$} & \multirow{2}{*}{$\begin{array}{l}\text { Site- } \\
\text { years } \\
\text { studied }^{4}\end{array}$} & \multirow{2}{*}{$\begin{array}{l}\text { Cropping } \\
\text { system }^{5}\end{array}$} & \multirow{2}{*}{$\begin{array}{l}\text { Yield } \\
\text { without } \\
\text { fertilizer } \mathrm{K}^{6}\end{array}$} & \multicolumn{2}{|c|}{ Yield response to $K^{7}$} & \multirow[b]{2}{*}{ Reference } \\
\hline & & & & & & & Lowest & Highest & \\
\hline & $\begin{array}{l}\text { Alfisols (sl) } \\
\text { Aridisols (sl) } \\
\text { Inceptisols (sl) } \\
\text { Inceptisols (sl) }\end{array}$ & $\begin{array}{l}125 \\
160(15) \\
138 \\
75^{8}\end{array}$ & $\begin{array}{l}0-156 \\
0-75 \\
0-166 \\
62-187\end{array}$ & $\begin{array}{l}3 \\
3 \\
4 \\
8\end{array}$ & $\begin{array}{l}\frac{\mathrm{Sc}}{9}^{9} \\
\frac{\mathrm{W}}{\mathrm{w}}^{9} \\
\frac{\mathrm{Po}^{9}}{\mathrm{Po}^{9}}\end{array}$ & $\begin{array}{l}103.6 \\
4.7 \\
28.4 \\
21.3^{10}\end{array}$ & $\begin{array}{l}11 *(125) \\
10^{*}(50) \\
17 *(42) \\
17 *(124)\end{array}$ & $\begin{array}{l}19 *(188) \\
14 *(75) \\
38 *(166) \\
21 *(187)\end{array}$ & $\begin{array}{l}180 \\
181 \\
182 \\
183\end{array}$ \\
\hline Indonesia & $\begin{array}{l}\text { Oxisols } \\
\text { Oxisols } \\
\text { Ultisols (c) }\end{array}$ & $\begin{array}{l}51^{8} \\
\mathrm{NR} \\
55^{8}(12)\end{array}$ & $\begin{array}{l}0-75 \\
0-105 \\
23-83\end{array}$ & $\begin{array}{l}1 \\
1 \\
6\end{array}$ & $\begin{array}{l}\overline{\frac{C}{P}} \\
\frac{\mathrm{C} p-R i-S^{9}}{}\end{array}$ & $\begin{array}{l}0.5 \\
0.2 \\
6.3^{10}\end{array}$ & $\begin{array}{l}400 *(25) \\
1^{\text {ns }}(26) \\
37 *(83)\end{array}$ & $\begin{array}{l}600 *(75) \\
11 *(105) \\
37 *(83)\end{array}$ & $\begin{array}{l}184 \\
185 \\
105\end{array}$ \\
\hline Pakistan & $\begin{array}{l}\text { Aridisols (cl) } \\
\text { Aridisols (sicl) }\end{array}$ & $\begin{array}{l}135^{8}(15) \\
160^{8}(15) \\
125^{8}(25)\end{array}$ & $\begin{array}{l}0-148 \\
0-148 \\
0-199\end{array}$ & $\begin{array}{l}1 \\
1 \\
1\end{array}$ & $\begin{array}{l}\mathrm{Ri}-\mathrm{W}_{\mathrm{w}}{ }^{9} \\
\mathrm{~F}-\overline{\mathrm{W}}_{\mathrm{w}}{ }^{9} \\
\mathrm{~W}_{\mathrm{w}}{ }^{9}\end{array}$ & $\begin{array}{l}2.5 \\
2.1 \\
3.6\end{array}$ & $\begin{array}{c}-14^{\mathrm{ns}}(111) \\
-12^{*}(148) \\
11^{\mathrm{ns}}(33)\end{array}$ & $\begin{array}{l}23^{*}(37) \\
16^{*}(37) \\
29^{* *}(133-199)\end{array}$ & $\begin{array}{l}186 \\
187\end{array}$ \\
\hline $\begin{array}{l}\text { Turkey } \\
\text { Vietnam } \\
\text { Australasia }\end{array}$ & $\begin{array}{l}\text { Vertisols (c) } \\
\text { Ultisols }\end{array}$ & $\begin{array}{l}560-750^{8} \\
8^{8}(20)\end{array}$ & $\begin{array}{l}0-199 \\
0-133\end{array}$ & $\begin{array}{l}2 \\
9\end{array}$ & $\overline{\overline{\mathrm{Ct}}}$ & $\begin{array}{l}2.6 \\
2.8\end{array}$ & $\begin{array}{l}12 *(66) \\
454 *(33)\end{array}$ & $\begin{array}{l}27 *(133) \\
546^{*}(133)\end{array}$ & $\begin{array}{l}188 \\
189\end{array}$ \\
\hline Australia & $\begin{array}{l}\text { Alfisols }_{4}, \text { Entisols }_{5} \\
\text { Inceptisols }_{1} \text { (s) }\end{array}$ & $19-37^{8}(10)$ & $0-60$ & 10 & $\underline{\mathrm{Cn}}$ & 1.(2) & $25 *(15)$ & $51 *(60)$ & 190 \\
\hline Mauke & Ultisols (cl) & NR & $0-132$ & 1 & Pn & 1.2 & $-16^{*}(132)$ & $-16 *(132)$ & 191 \\
\hline
\end{tabular}

$* * *, * * *$ Significant at $\alpha=0.05,0.01$ and 0.001 , respectively; ns, not significant.

${ }^{1}$ A complete listing of all studies surveyed can be found in Table S4, for the online version of the paper.

2 Surface texture designated parenthetically as c (clay), cl (clay loam), fs (fine sand), fsl (fine sandy loam), gl (gravelly loam), 1 (loam), lfs (loamy fine sand), ls (loamy sand), s (sand), scl (sandy clay loam), si (silt), sicl (silty clay loam), sil (silt loam) and sl (sandy loam). Subscripted values indicate the number of soils studied that represent a specific order.

3 Air-dried data reported. Values in parentheses indicate sampling depth in $\mathrm{cm}$. NR, not reported.

${ }^{4}$ Values take into account the number of genotypes studied and/or the use of multiple tillage systems.

5 A, alfalfa (Medicago sativa L.); B, barley (Hordeum vulgare L.); Bg, bermudagrass (Cynodon dactylon L. Pers.); Bt, birdsfoot trefoil (Lotus corniculatus L.); C, corn (Zea mays L.); Cn, canola (Brassica napus and campestris); Cp, cowpea (Vigna unguiculata L.); Cs, cassava (Manihot esculenta); Ct, cotton (Gossypium hirsutum L.); F, fallow; Gn, groundnut (Arachis hypogoea L.);

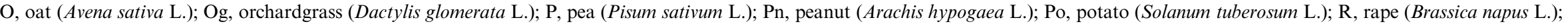
$\mathrm{Ra}$, radish (Raphanus sativus L.); Rc, reed canarygrass (Phalaris arundinacea L.); Rcl, red clover (Trifolium pratense L.); Ri, rice (Oryza sativa L.); S, soybean (Glycine max L. Merr.);

$\mathrm{Sa}$, safflower (Carthamus tinctorius L.); Sb, sugarbeet (Beta vulgaris L.); Sbg, smooth bromegrass (Bromus inermis Leyss.); Sc, sugarcane (Saccharum officinarum L.); Sg, small grain; Tf, tall

fescue (Festuca arundinacea Schreb.); Vg, vegetables; Wc, white clover (Trifolium repens $\mathrm{L}$ ).; $\mathrm{W}_{\mathrm{w}}$, winter wheat (Triticum aestivum $\mathrm{L}$.). Underscoring indicates the crop(s) studied for $\mathrm{K}$ response.

${ }^{6}$ Data for legumes were obtained from fertilization with $\mathrm{P}$ but not $\mathrm{K}$, whereas data for non-legumes were obtained from fertilization with $\mathrm{N}$ or $\mathrm{N}$ and $\mathrm{P}$ but not $\mathrm{K}$. Parentheses indicate digital uncertainty in estimating data reported in figures. When otherwise reported, grain yields were adjusted to market-standard moisture content.

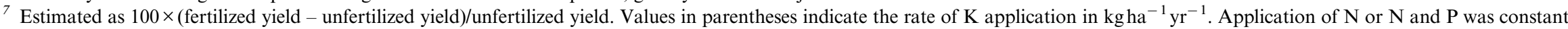
throughout the range of $\mathrm{K}$ rates cited.

${ }^{8}$ Values expressed as $\mathrm{mg} \mathrm{kg}^{-1}$

9 Above-ground residues removed.

${ }^{10}$ Yield when the lowest $\mathrm{K}$ rate was applied with $\mathrm{N}$ or $\mathrm{N}$ and $\mathrm{P}$. 
Many leguminous crops are sensitive to $\mathrm{Cl}^{-}$toxicity, including soybean ${ }^{120,131}$ and alfalfa ${ }^{159,160,198}$, and $\mathrm{Cl}^{-}$ can reduce soil $\mathrm{N}$ availability by inhibiting nitrification in soils ${ }^{195,196,199,200}$ (see also supplementary references [53-55] for the online version of the paper) and by acting as a competitive anion that suppresses plant uptake of $\mathrm{NO}_{3}^{-}$147,201,202 (see also supplementary references [38] and [56-59] for the online version of the paper). A further difficulty arises from the mobility of $\mathrm{Cl}^{-}$in soils, which intensifies profile leaching of $\mathrm{Ca}^{2+}$ as a counterion ${ }^{203-205}$.

Producers have long been led to believe that $\mathrm{KCl}$ fertilization serves an essential role, not only for sustaining crop yield but more importantly, for ensuring a highquality product that will maximize economic return. To ascertain the credibility of the latter claim, a thorough survey was undertaken of peer-reviewed and university publications that provide the most reliable source of information regarding the agronomic effects of $\mathrm{KCl}$. The findings, summarized in Table 5 for more than 1000 field experiments, altogether contradict the prevailing belief in the value of this fertilizer for improving crop quality, since the frequency of positive responses was only about $8 \%$. On the contrary, the qualitative effect of $\mathrm{KCl}$ was negative in $57 \%$ of the trials surveyed. In some of these trials, crop quality was reduced despite a significant yield increase $\mathrm{i}^{159,160}$.

One of the better known consequences of $\mathrm{KCl}$ fertilization, reported in Table 5 for studies with corn $^{153,201,212,216}$, wheat ${ }^{221}$ and sorghum (Sorghum bicolor L. Moench) $)^{137}$, arises from the antagonistic effect of $\mathrm{Cl}^{-}$on $\mathrm{NO}_{3}^{-}$uptake that reduces lodging or stalk rot when susceptible cereal varieties are grown on a soil with high $\mathrm{N}$-supplying power and/or with heavy inputs of fertilizer $\mathrm{N}$, but at the expense of promoting $\mathrm{NO}_{3}^{-}$loss through leaching or denitrification. The use of $\mathrm{KCl}$ can also have a beneficial effect by increasing the fiber strength (micronaire) of cotton (Gossypium hirsutum L.) grown on soils inherently limited in K reserves ${ }^{222-224,226}$, which would be expected considering the lack of a dense rooting system or residue $\mathrm{K}$ inputs.

Other qualitative benefits are often claimed for $\mathrm{KCl}$ fertilization of grain, fiber, oilseed or sugar crops, but these are difficult to reconcile with the listings in Table 5. This fertilizer, for example, has usually been ineffective for reducing disease severity in barley (Hordeum vulgare L.), wheat, soybean or cotton, and even with a positive effect, there was no significant yield increase ${ }^{202,210,219,225}$ except where root rot was associated with a high content of $\mathrm{NO}_{3}^{-}$but not $\mathrm{K}^{+}$or $\mathrm{Cl}^{-147}$ or when a susceptible variety was grown on an infertile sand ${ }^{207}$. There was likewise little evidence that $\mathrm{KCl}$ enhances sucrose content in sugar crops or oil, protein or fiber content or composition. Instead, these constituents were more likely to be adversely affected, as reported by Fintel and Quicke ${ }^{214}$ for corn protein that declined in niacin and tryptophan. Such a decline has serious implications for human nutrition in the developing world, where grain is the staple food source.
Tuber crops are no less important to human nutrition, and particularly potato (Solanum tuberosum $\mathrm{L}$.) that has a much higher $\mathrm{K}$ requirement than grain crops. In many parts of the world, $\mathrm{KCl}$ fertilization is a normal practice for potato production; however, there is a hidden cost from lowering starch content, which in turn reduces specific gravity. These effects, clearly documented in Table 5, have adverse consequences for human health such as obesity and cardiovascular disease, arising from greater oil retention in processed products such as potato chips and french fries. The anion supplied by $\mathrm{KCl}$ intensifies the decline in starch or dry matter content and specific gravity ${ }^{163,238,241-244}$, and becomes a toxicological concern by enhancing the mobility and plant uptake of soil $\mathrm{Cd}^{239,240,247}$. The latter problem has also been reported for cereal crops ${ }^{208,220}$, and may thus be of broader interest for contamination of the food chain, particularly in view of recent clinical evidence linking breast cancer to dietary Cd exposure ${ }^{248}$.

Forage crops remove large amounts of $\mathrm{K}$ because their aboveground biomass is repeatedly harvested, and intensive usage of fertilizer $\mathrm{KCl}$ is widely promoted to not only sustain productivity, but more importantly, to ensure high forage quality that is critical to animal nutrition. Unfortunately, Table 5 provides no evidence that $\mathrm{KCl}$ is of any qualitative benefit to forage crops; rather, the effect was invariably negative, often involving a low content of $\mathrm{Ca}$ and/or $\mathrm{Mg}^{159-161,229,230}$ that predisposes livestock to milk fever ${ }^{249,250}$ or grass tetany ${ }^{229,251}$. To prevent these disorders while improving forage quality, Cherney et al. ${ }^{252}$ have recommended that the use of fertilizer $\mathrm{K}$ or manure be avoided on dairy farms in the northern USA, unless $\mathrm{K}$ deficiency is detected by plant analysis.

Elevated $\mathrm{K}$ supplies have long been recognized to have an antagonistic impact on the bioavailability of $\mathrm{Ca}$ and $\mathrm{Mg}$, with adverse consequences for crop yield and quality. For example, McCalla ${ }^{253}$ found that raising the $\mathrm{K}: \mathrm{Ca}$ ratio in culture studies led to a progressive decrease in the growth of legume bacteria and in the nodulation of alfalfa and soybean roots. This imbalance would normally be ameliorated by liming, but even a neutral Ca salt can be utilized successfully to enhance soybean nodulation on an acidic soil ${ }^{254}$. There are broader ramifications from the antagonistic effect of $\mathrm{K}$ on the Ca content of food crops, because low dietary $\mathrm{Ca}$ intake has been linked to several human diseases, including osteoporosis, rickets and colon cancer $^{255-259}$.

The problems associated with $\mathrm{KCl}$ usage have been thoroughly documented for arid and semi-arid regions by Krauss and Saurat ${ }^{260}$, and arise in part from the presence of a bioactive anion that has detrimental consequences for crop yield and quality. Some of these consequences can be avoided by applying $\mathrm{K}_{2} \mathrm{SO}_{4}$ instead of $\mathrm{KCl}$. The benefits of this strategy for increasing crop yield and/or quality have been documented in numerous studies with potato ${ }^{133,163,168,261-265}$ and to a lesser extent 


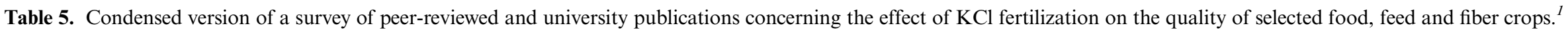

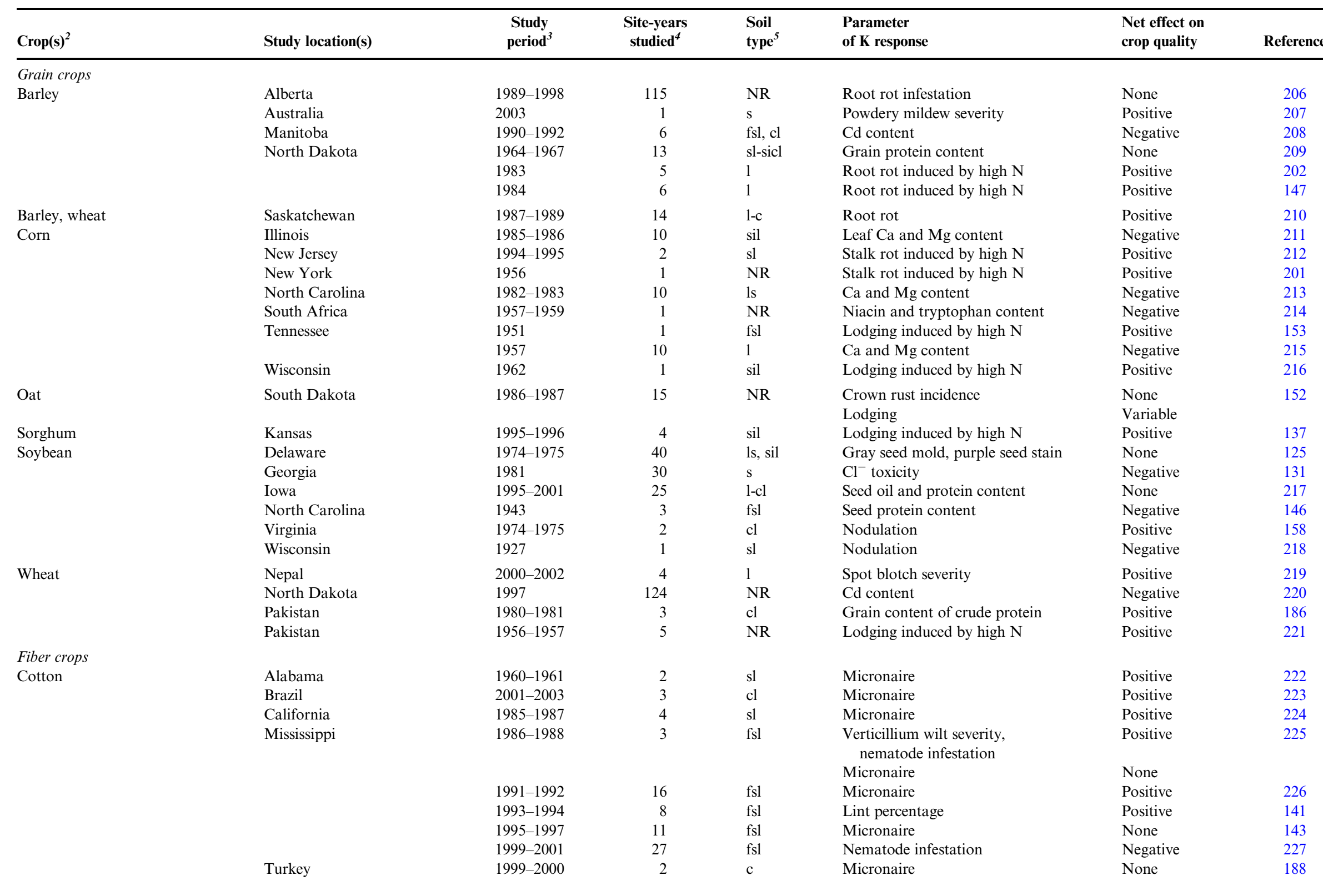


Mixed grass-clover

Australia

Red clover

Wisconsin

1966-1971

Sorghum $\times$ sudangrass

New York

1974

Australia

Subterranean clover-ryegrass

Tall fescue

West Virginia

1968-1973

1971-1975

1973-1986

Peanut

Alabama

Australia

India

1993-2003

1969-1970

Safflower

Sunflower

Bangladesh

1973-1974

Minnesota, North Dakota

NR

Sugar crops

Sugarbeet

Sugarcane

England

Brazil

Florida

India

2006

1970-1972

1989-1992

Australia

Potato

Idaho

India

1982-1984

1992-1993

1991-1992

1989-1999

970-1976

NR

2001-2002

2000-2001

1961-1966

1995-1996

Macedonia

$1955-1958$

Maine

1964-1967

1988-1989

1992-1995

Utah

Wisconsin

Sweet potato

Louisiana

1968-1975
$\mathrm{Ca}$ and $\mathrm{Mg}$ content

$\mathrm{Cl}^{-}$toxicity

$\mathrm{Ca}$ and $\mathrm{Mg}$ conten

$\mathrm{Ca}$ and $\mathrm{Mg}$ content

Incidence of grass tetany

$\mathrm{Ca}$ and $\mathrm{Mg}$ conten

$\mathrm{Ca}$ and $\mathrm{Mg}$ and crude protein

content, milk production

Mg content

$\mathrm{Ca}$ and $\mathrm{Mg}$ content

Kernel grade

Seed oil content

Seed protein and oil content

Seed protein content

Seed oil content

Cd content

Negative

Negative

Negative

Negative

Negative

Negative

Negative

Negative

Negative

None

None

Negative

Negative

Positive

Negative

Sucrose content, juice purity

None

Sucrose conten

Sucrose content

Sucrose content

Sucrose yield

None

None

Negative

Positive

Negative

gravity

Cd content

Cd content

Dry matter content

Specific gravity

Specific gravity

Dry matter content,

specific gravity, chip color

Tuber weight

Dry matter content

Starch content

Specific gravity

Dry matter content

Specific gravity

Specific gravity

Dry matter and protein

content, firmness

${ }^{1}$ A complete listing of all studies surveyed can be found in Table S5 for the online version of the paper.

2 Latin names for crops not included in Table 4: ryegrass, Lolium perenne L. and Lolium rigidum Gaudin; subterranean clover, Trifolium subterranean L.

${ }^{3} \mathrm{NR}$, not reported

${ }^{4}$ Values take into account number of genotypes studied.

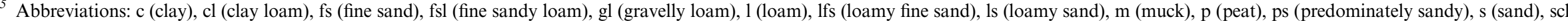

(sandy clay loam), sic (silty clay), sicl (silty clay loam), sil (silt loam), sl (sandy loam). NR, not reported. 
with several other crops, including alfalfa ${ }^{160}$, soybean ${ }^{266}$ and wheat ${ }^{186,267}$.

Tables 4 and 5 (see also Tables S4 and S5 for the online version of the paper) provide little agronomic justification for the buildup-maintenance philosophy that for several decades has intensified the input of fertilizer $\mathrm{K}$, often in conjunction with direct or indirect subsidies at considerable public expense ${ }^{268,269}$ (see also supplemental references [60-62] for the online version of the paper). The result has been massive $\mathrm{KCl}$ consumption, which for Illinois has surpassed $21 \mathrm{Tg}$ since 1950 , equivalent on average to approximately $2200 \mathrm{~kg} \mathrm{ha}^{-1270-272}$. A cumulative effect on soil physical and chemical properties would be expected, since $\mathrm{K}$ is prone to interlayer fixation that collapses 2:1 clay minerals and converts an active, swelling smectite to an inactive, non-swelling illite ${ }^{26,273}$. The stabilizing value of $\mathrm{KCl}$ has long been recognized in the construction of impervious pavement and foundations $^{274-277}$. Unfortunately, the agronomic consequences include a loss of $\mathrm{CEC}^{71,77,278-280}$ and lower water-holding capacity, which is not conducive to crop growth and productivity.

\section{Conclusions}

Since the onset of industrialized agriculture more than half a century ago, the view has been inculcated that intensive inputs of fertilizer $\mathrm{K}$ are indispensible for maximizing crop yield and quality and for the long-term maintenance of soil productivity. This view cannot be reconciled with the considerable volume of scientific evidence presented herein, encompassing soil testing for plant-available $\mathrm{K}$ and the consequences of $\mathrm{KCl}$ fertilization for agricultural productivity, food safety and soil degradation.

If fertilizer $\mathrm{K}$ usage is to be profitable in a production setting, current recommendations will no longer suffice that rely on soil testing for exchangeable K. As a more viable alternative, producers should periodically carry out their own strip trials, for comparing yield with and without upward and downward $\mathrm{K}$ rate adjustment. Initially, a 3-year period would be appropriate for repeating these trials, but a longer interval could safely be employed with cash-grain cropping that limits $\mathrm{K}$ removal. To avoid the adverse consequences of $\mathrm{Cl}^{-}$, $\mathrm{K}_{2} \mathrm{SO}_{4}$ would be preferred as a fertilizer source.

Acknowledgements. This study was performed with partial funding under TIPAN Project 391-0488, and with additional funding through the ${ }^{15} \mathrm{~N}$ Analysis Service and Projects ILLU875-316 and ILLU-875-375, Illinois Agricultural Experiment Station. We thank Dr T.R. Peck (now deceased) for directing a thesis project by the senior author to quantify soil $\mathrm{K}$ dynamics in relation to fertility evaluation and fertilizer recommendations, and for preserving and archiving soil samples collected from the Morrow Plots. Appreciation is also expressed to the numerous scientists whose work is cited in Tables 2, 4 and 5; to all responsible for cropping the Morrow Plots since 1876; and to library personnel at the University of Illinois who assisted in obtaining many references cited. Finally, we acknowledge Dr C.G. Hopkins for recognizing a century ago the importance of profile $\mathrm{K}$ reserves for sustaining crop $\mathrm{K}$ nutrition.

\section{References}

1 Howard, A. 1947. The Soil and Health. A Study of Organic Agriculture. Devin-Adair, New York.

2 Snow, J.S. 2009. Misunderstanding Soil Ecosystems. MA thesis, Tufts University, Boston, MA. Available at Web site http://www.tufts.edu/water/pdf/Thesis/JSnowThesis.pdf (verified November 27, 2012).

3 Visser, J. 2010. Down to Earth. PhD thesis, Wageningen University, Wageningen, The Netherlands. Available at Web site http://edepot.wur.n1/135448 (verified November 27, 2012).

4 Truog, E. 1938. Putting soil science to work. Journal of the American Society of Agronomy 30:973-985.

5 Liebhardt, W.C. and Cotnoir, L. 1979. Potassium fertilizer recommendations and changes in potassium soil test values as influenced by additions of potassium. Communications in Soil Science and Plant Analysis 10:831-840.

6 Olson, R.A., Anderson, F.N., Frank, K.D., Grabouski, P.H., Rehm, G.W., and Shapiro, C.A. 1987. Soil testing interpretations: Sufficiency vs. build-up and maintenance. In J.R. Brown (ed.). Soil Testing: Sampling, Correlation, Calibration, and Interpretation. Soil Science Society of America, Madison, WI. p. 41-52.

7 Thomas, G.W. 1989. The soil bank account and the farmer's bank account. Journal of Production Agriculture 2:122-124.

8 Hopkins, C.G. and Aumer, J.P. 1915. Potassium from the Soil. Illinois Agricultural Experiment Station Bulletin 182. University of Illinois, Urbana, IL.

9 Blair, A.W. 1919. Utilizing soil potash by means of intermediary crops. Proceedings of the Society for Promotion of Agricultural Science 39:69-74.

10 Reitemeier, R.F. 1951. Soil potassium. Advances in Agronomy 3:113-164.

11 Srinivasa Rao, C., Rupa, T.R., Rao, A.S., and Bansal, S.K. 2001. Subsoil potassium availability in twenty-two benchmark soil series of India. Communications in Soil Science and Plant Analysis 32:863-876.

12 Bradfield, R. 1946. Where are the new discoveries in soil science leading? I. The physical chemistry of soil-plant relationships. Soil Science Society of America Proceedings 11:3-8.

13 Bray, R.H. 1944. Soil-plant relations I. The quantitative relation of exchangeable potassium to crop yields and to crop response to potash additions. Soil Science 58:305-324.

14 Bear, F.E. and Toth, S.J. 1948. Influence of calcium on availability of other soil cations. Soil Science 65:69-74.

15 Eckert, D.J. 1987. Soil test interpretations: Basic cation saturation ratios and sufficiency levels. . In J.R. Brown (ed.). Soil Testing: Sampling, Correlation, Calibration, and Interpretation. Soil Science Society of America, Madison, WI. p. 53-64.

16 Kopittke, P.M. and Menzies, N.W. 2007. A review of the use of the basic cation saturation ratio and the 'ideal' soil. Soil Science Society of America Journal 71:259-265. 
17 Bray, R.H. and DeTurk, E.E. 1938. The release of potassium from non-replaceable forms in Illinois soils. Soil Science Society of America Proceedings 3:101-106.

18 Luebs, R.E., Stanford, G., and Scott, A.D. 1956. Relation of available potassium to soil moisture. Soil Science Society of America Proceedings 20:45-50.

19 Martin, H.W. and Sparks, D.L. 1985. On the behavior of nonexchangeable potassium in soils. Communications in Soil Science and Plant Analysis 16:133-162.

20 Mengel, K. and Uhlenbecker, K. 1993. Determination of available interlayer potassium and its uptake by ryegrass. Soil Science Society of America Journal 57:761-766.

21 Skogley, E.O. 1994. Reinventing soil testing for the future. In J.L. Havlin and J.S. Jacobsen (eds). Soil Testing: Prospects for Improving Nutrient Recommendations. Soil Science Society of America Special Publication 40. Soil Science Society of America, Madison, WI. p. 187201.

22 Khan, S.A. 1991. Soil K level in relation to K treatment, season of year and soil moisture level. PhD thesis, University of Illinois, Urbana, IL.

23 Wascher, H.L., Alexander, J.D., Ray, B.W., Beavers, A.H., and Odell, R.T. 1960. Characteristics of Soils Associated with Glacial Tills in Northeastern Illinois. Illinois Agricultural Experiment Station Bulletin 665. University of Illinois, Urbana, IL.

24 Knudsen, D., Peterson, G.A., and Pratt, P.F. 1982. Lithium, sodium, and potassium. In A.L. Page, R.H. Miller, and D.R. Keeney (eds). Methods of Soil Analysis. Part 2. Chemical and Microbiological Properties. Agronomy Monograph 9. 2nd ed. American Society of Agronomy and Soil Science Society of America, Madison, WI. p. 225246.

25 Suhr, N.H. and Ingamells, C.O. 1966. Solution technique for analysis of silicates. Analytical Chemistry 38:730-734.

26 Velde, B. and Peck, T. 2002. Clay mineral changes in the Morrow experimental plots, University of Illinois. Clays and Clay Minerals 50:364 -370 .

27 Khan, S.A., Mulvaney, R.L., Ellsworth, T.R., and Boast, C.W. 2007. The myth of nitrogen fertilization for soil carbon sequestration. Journal of Environmental Quality 36:18211832.

28 Fernández, F.G. and Hoeft, R.G. 2009. Managing soil $\mathrm{pH}$ and crop nutrients. In E. Nafziger (ed.) Illinois Agronomy Handbook. 24th ed. University of Illinois, Urbana, IL. p. 91-112.

29 Leask, W.C. and Daynard, T.B. 1973. Dry matter yield, in vitro digestibility, percent protein, and moisture of corn stover following grain maturity. Canadian Journal of Plant Science 53:515-522.

30 Kiniry, J.R., Williams, J.R., Vanderlip, R.L., Atwood, J.D., Reicosky, D.C., Mulliken, J., Cox, W.J., Mascagni, H.J. Jr, Hollinger, S.E., and Wiebold, W.J. 1997. Evaluation of two maize models for nine U.S. locations. Agronomy Journal 89:421-426.

31 Prince, S.D., Haskett, J., Steininger, M., Strand, H., and Wright, R. 2001. Net primary production of U.S. Midwest croplands from agricultural harvest yield data. Ecological Applications 11:1194-1205.

32 Tollenaar, M., Ahmadzadeh, A., and Lee, E.A. 2004. Physiological basis of heterosis for grain yield in maize. Crop Science 44:2086-2094.
33 Mengel, K. 2007. Potassium. In A.V. Barker and D.J. Pilbeam (eds). Handbook of Plant Nutrition. Taylor \& Francis, Boca Raton, FL. p. 91-120.

34 Lovell, M.C. 2012. Seasonal adjustment of economic time series and multiple regression analysis. Journal of the American Statistical Association 58:993-1010.

35 SAS Institute. 2013. SAS Onlinedoc 9.1.3. SAS Institute, Cary, NC. Available at Web site http://support. sas.com/onlinedoc/913/docMainpage.jsp (verified March 21, 2013).

36 Hochberg, Y. 1988. A sharper Bonferroni procedure for multiple tests of significance. Biometrika 75:800-802.

37 Attoe, O.J. 1946. Potassium fixation and release in soils occurring under moist and drying conditions. Soil Science Society of America Proceedings 11:145-149.

38 Hanway, J.J., Barber, S.A., Bray, R.H., Caldwell, A.C., Fried, M., Kurtz, L.T., Lawton, K., Pesek, J.T., Pretty, K., Reed, M., and Smith, F.W. 1962. North Central Regional Potassium Studies III. Field Studies with Corn. Iowa Agricultural Experiment Station Research Bulletin 503. Iowa State University, Ames, IA.

39 Hoskinson, R.L., Hess, J.R., and Alessi, R.S. 1999. Temporal changes in the spatial variability of soil nutrients. In J.V. Stafford (ed.). Precision Agriculture '99. Part 1. Academic Press, Sheffield, England. p. 61-70.

40 Blakemore, M. 1966. Seasonal changes in the amounts of phosphorus and potassium dissolved from soils by dilute calcium chloride solutions. Journal of Agricultural Science, Cambridge 66:139-146.

41 Childs, F.D. and Jencks, E.M. 1967. Effect of time and depth of sampling upon soil test results. Agronomy Journal 59:537-540.

42 International Plant Nutrition Institute. 2009. Soil Fertility Shifts in Eastern Canada and the Northeastern USA. International Plant Nutrition Institute, Norcross, GA. Available at Web site http://nane.ipni.net/articles/ NANE0047-EN (verified May 25, 2012).

43 Fixen, P.E. 2006. Soil test levels in North America. Better Crops 90(1):4-7. Available at Web site http://www.ipni.net/ publication/bettercrops.nsf/0/1403E75A034B3F3A85257980 00771851/\$FILE/Better $\% 20$ Crops $\% 202006-1 \% 20$ (lo\%20res). pdf (verified August 13, 2012).

44 Cope, J.T. Jr 1970. Response of Cotton, Corn, Bermudagrass to Rates of N, P, and K. Alabama Agricultural Experiment Station Circular 181. Auburn University, Auburn, AL.

45 Cope, J.T. Jr 1981. Effects of 50 years of fertilization with phosphorus and potassium on soil test levels and yields at six locations. Soil Science Society of America Journal 45:342-347.

46 Cope, J.T. Jr 1984. Long-Term Fertility Experiments on Cotton, Corn, Soybeans, Sorghum, and Peanuts, 1929-1982. Alabama Agricultural Experiment Station Bulletin 561. Auburn University, Auburn, AL.

47 Mainz, M.A. 1987. Potassium rate study. In Report of Research Results, Northwestern Illinois Agricultural Research and Demonstration Center. University of Illinois, Urbana, IL. p. 45-48.

48 Ebelhar, S.A. and Varsa, E.C. 1999. Tillage and potassium placement effects on potassium use efficiency in a corn-soybean rotation. In R.G. Hoeft (ed.). 1999 Illinois Fertilizer Conference Proceedings. University of Illinois, 
Urbana, IL. p. 161-180. Available at Web site http:// frec.ifca.com/1999/report13/index.htm (verified August 9, 2012)

49 Li, R.-G. and Barber, S.A. 1988. Effect of phosphorus and potassium fertilizer on crop response and soil fertility in a long-term experiment. Fertilizer Research 15:123-136.

50 Mallarino, A.P., Webb, J.R., and Blackmer, A.M. 1991. Soil test values and grain yields during 14 years of potassium fertilization of corn and soybean. Journal of Production Agriculture 4:562-566.

51 Mallarino, A.P., Webb, J.R., and Blackmer, A.M. 1991. Corn and soybean yields during 11 years of phosphorus and potassium fertilization on a high-testing soil. Journal of Production Agriculture 4:312-317.

52 Vitosh, M.L., Lucas, R.E., and Silva, G.H. 1997. Long-term effects of fertilizer and manure on corn yield, soil carbon, and other soil chemical properties in Michigan. In E.A. Paul, K.A. Paustian, E.T. Elliott and C.V. Cole (eds). Soil Organic Matter in Temperate Agroecosystems: Long-Term Experiments in North America. CRC Press, Boca Raton, FL. p. 129-139.

53 Randall, G.W., Evans, S.D., and Iragavarapu, T.K. 1997. Long-term $\mathrm{P}$ and $\mathrm{K}$ applications. II. Effect on corn and soybean yields and plant $\mathrm{P}$ and $\mathrm{K}$ concentrations. Journal of Production Agriculture 10:572-580.

54 Randall, G.W., Iragavarapu, T.K., and Evans, S.D. 1997. Long-term $\mathrm{P}$ and $\mathrm{K}$ applications: I. Effect on soil test incline and decline rates and critical soil test levels. Journal of Production Agriculture 10:565-571.

55 Brown, J.R., Osburn, D.D., Redhage, D., and Gantzer, C.J. 1995. Multi-crop comparisons on Sanborn Fields, Missouri, USA. In V. Barnett, R. Payne and R. Steiner (eds). Agricultural Sustainability. Economic, Environmental and Statistical Considerations. John Wiley, Chichester, England. p. 111-132.

56 Olson, R.A., Frank, K.D., Grabouski, P.H., and Rehm, G.W. 1982. Economic and agronomic impacts of varied philosophies of soil testing. Agronomy Journal 74:492-499.

57 Markus, D.K. and Battle, W.R. 1965. Soil and plant responses to long-term fertilization of alfalfa (Medicago sativa L.). Agronomy Journal 57:613-616.

58 Woodhouse, W.W. Jr 1968. Long-term fertility requirements of coastal bermudagrass. I. Potassium. Agronomy Journal 60:508-512.

59 Davis, R.L., Patton, J.J., Teal, R.K., Tang, Y., Humphreys, M.T., Mosali, J., Girma, K., Lawles, J.W., Moges, S.M., Malapati, A., Si, J., Zhang, H., Deng, S., Johnson, G.V., Mullen, R.W., and Raun, W.R. 2003. Nitrogen balance in the Magruder Plots following 109 years in continuous winter wheat. Journal of Plant Nutrition 26:1561-1580.

60 Girma, K., Holtz, S.L., Arnall, D.B., Tubaña, B.S., and Raun, W.R. 2007. The Magruder Plots: Untangling the puzzle. Agronomy Journal 99:1191-1198.

61 Harper, H.J. 1959. 65 Years of Continuous Wheat. Bulletin B-531. Oklahoma State University, Stillwater, OK.

62 Lutz, J.A. Jr 1973. Potassium supplying power of Groseclose silt loam for corn. Communications in Soil Science and Plant Analysis 4:23-30.

63 Zhang, H., Wang, B., and Xu, M. 2008. Effects of inorganic fertilizer inputs on grain yields and soil properties in a longterm wheat-corn cropping system in south China.
Communications in Soil Science and Plant Analysis 39:1583-1599.

64 Zhang, H.-M., Wang, B.-R., Xu, M.-G., and Fan, T.-L. 2009. Crop yield and soil responses to long-term fertilization on a red soil in southern China. Pedosphere 19:199-207.

65 Wang, X., Hoogmoed, W.B., Cai, D., Perdok, U.D., and Oenema, O. 2007. Crop residue, manure and fertilizer in dryland maize under reduced tillage in northern China: II Nutrient balances and soil fertility. Nutrient Cycling in Agroecosystems 79:17-34.

66 Wang, X., Cai, D., Hoogmoed, W.B., Perdok, U.D., and Oenema, O. 2007. Crop residue, manure and fertilizer in dryland maize under reduced tillage in northern China: I Grain yields and nutrient use efficiencies. Nutrient Cycling in Agroecosystems 79:1-16.

67 Johnston, A.E. and Poulton, P.R. 1977. Yields on the Exhaustion Land and changes in the NPK content of the soils due to cropping and manuring, 1852-1975. In Rothamsted Experimental Station Report for 1976. Part 2. Lawes Agricultural Trust, Harpenden, Herts, England. p. 53-85.

68 Last, P.J., Webb, D.J., Bugg, R.B., Bean, K.M.R., Durrant, M.J., and Jaggard, K.W. 1985. Long-term effects of fertilizers at Broom's Barn, 1965-82. In Rothamsted Experimental Station Report for 1984. Part 2. Lawes Agricultural Trust, Harpenden, Herts, England. p. 231-249.

69 Blake, L., Mercik, S., Koerschens, M., Goulding, K.W.T., Stempen, S., Weigel, A., Poulton, P.R., and Powlson, D.S. 1999. Potassium content in soil, uptake in plants and the potassium balance in three European long-term field experiments. Plant and Soil 216:1-14.

70 Dam Kofoed, A. and Nemming, O. 1976. Askov 1894: Fertilizers and manure on sandy and loamy soils. Annales Agronomiques 27:583-610.

71 Amberger, A. and Gutser, R. 1976. Effect of long-term potassium fertilization on crops and potassium dynamics of a brown earth (Weihenstephan). Annales Agronomiques 27:643-657.

72 Stumpe, H., Garz, J., Johannemann, R., and Buchte, M. 1989. Einfluß der K-Düngung auf den Ertrag und einige Bodeneigenschaften in einem Dauerversuch auf einer Sandlöß-Braunschwarzerde in Halle. Archiv für Ackerund Pflanzenbau und Bodenkunde 33:301-310.

73 Brar, B.S. and Pasricha, N.S. 1998. Long-term studies on integrated use of organic and inorganic fertilizers in maizewheat-cowpea cropping system on alluvial soil of Punjab. In A. Swarup, D. Damodar Reddy, and R.N. Prasad (eds). Proceedings of a National Workshop on Long-term Soil Fertility Management Integrated Plant Nutrient Supply. Indian Institute of Soil Science, Bhopal, India. p. 154-168.

74 Sharma, S.P., Sharma, J., and Subehia, S.K. 1998. Longterm effects of chemical fertilizers on crop yields, nutrients uptake and soil environment in western Himalayan soils. In A. Swarup, D. Damodar Reddy, and R.N. Prasad (eds). Proceedings of a National Workshop on Long-term Soil Fertility Management Integrated Plant Nutrient Supply. Indian Institute of Soil Science, Bhopal, India. p. 125-138.

75 Lee, R. and Metson, A.J. 1977. Potassium removal from soil by lucerne over three years and the effect of potassium topdressing. New Zealand Journal of Agricultural Research 20:185-192. 
76 Agboola, A.A. 1970. Preliminary investigation on the effect of continuous cropping of maize on grain yield and on total nitrogen, available phosphorus and exchangeable potassium on three Nigerian soils. Nigerian Journal of Science 4:89-99.

77 De Datta, S.K., Gomez, K.A., and Descalsota, J.P. 1988. Changes in yield response to major nutrients and in soil fertility under intensive rice cropping. Soil Science 146:350-358.

78 Öborn, I., Edwards, A.C., and Hillier, S. 2010. Quantifying uptake rate of potassium from soil in a long-term grass rotation experiment. Plant and Soil 335:3-19.

79 Tisdale, S.L., Nelson, W.L., and Beaton, J.D. 1985. Soil Fertility and Fertilizers. 4th ed. Macmillan Publishing Company, New York, NY.

80 Cassman, K.G., Olk, D.C., Brouder, S.M., and Roberts, B. A. 1995. The influence of moisture regime, organic matter, and root eco-physiology on the availability and acquisition of potassium: Implications for tropical lowland rice. In Potassium in Asia. Balanced Fertilization to Increase and Sustain Agricultural Production. International Potash Institute, Basel, Switzerland. p. 135-156.

81 Abel, F.A.E. and Magistad, O.C. 1935. Conversion of soil potash from the non-replaceable to the replaceable form. Journal of the American Society of Agronomy 27:437-445.

82 Parker, D.R., Hendricks, G.J., and Sparks, D.L. 1989. Potassium in Atlantic Coastal Plain soils: II. Crop responses and changes in soil potassium under intensive management. Soil Science Society of America Journal 53:397-401.

83 Mengel, K., Rahmatullah, and Dou, H. 1998. Release of potassium from the silt and sand fraction of loess-derived soils. Soil Science 163:805-813.

84 Rouse, R.D. and Bertramson, B.R. 1949. Potassium availability in several Indiana soils: Its nature and methods of evaluation. Soil Science Society of America Proceedings 14:113-123.

85 Wood, L.K. and DeTurk, E.E. 1941. The absorption of potassium in soils in non-replaceable forms. Soil Science Society of America Proceedings 5:152-161.

86 Oliveira, V., Ludwick, A.E., and Beatty, M.T. 1971. Potassium removed from some southern Brazilian soils by exhaustive cropping and chemical extraction methods. Soil Science Society of America Proceedings 35:763-767.

87 Ganeshamurthy, A.N. 1983. An estimate of the uptake of subsurface soil potassium by crops in two long-term experiments. Journal of Agricultural Science, Cambridge 101:495-497.

88 Badraoui, M., Bloom, P.R., and Delmaki, A. 1992. Mobilization of non-exchangeable $\mathrm{K}$ by ryegrass in five Moroccan soils with and without mica. Plant and Soil 140:55-63.

89 Zubillaga, M.M. and Conti, M.E. 1996. Availability of exchangeable and non-exchangeable $\mathrm{K}$ in Argentine soils with different mineralogy. Zeitschrift für Pflanzenernährung und Bodenkunde 159:149-153.

90 Mortland, M.M., Lawton, K., and Uehara, G. 1958. Fixation and release of potassium by some clay minerals. Soil Science Society of America Proceedings 21:381-384.

91 Hinsinger, P.H., Dufey, J.E., and Jaillard, B. 1991. Biological weathering of micas in the rhizosphere as related to potassium absorption by plant roots. In B.L. McMichael and H. Persson (eds) Plant Roots and Their Environment. Elsevier, Amsterdam, The Netherlands. p. 98-105.
92 Hoffland, E., Kuyper, T.W., Wallander, H., Plassard, C., Gorbushina, A.A., Haselwandter, K., Holmström, S., Landeweert, R., Lundström, U.S., Rosling, A., Sen, R., Smits, M.M., van Hees, P.A.W., and van Breemen, N. 2004. The role of fungi in weathering. Frontiers in Ecology and the Environment 2:258-264.

93 van Schöll, L., Kuyper, T.W., Smits, M.M., Landeweert, R., Hoffland, E., and van Breemen, N. 2008. Rock-eating mycorrhizas: Their role in plant nutrition and biogeochemical cycles. Plant and Soil 303:35-47.

94 Arocena, J.M., Velde, B., and Robertson, S.J. 2012. Weathering of biotite in the presence of arbuscular mycorrhizae in selected agricultural crops. Applied Clay Science 64:12-17.

95 Yuan, T.L., Zelazny, L.W., and Ratanaprasatporn, A. 1976. Potassium status of selected Paleudults in the Lower Coastal Plain. Soil Science Society of America Journal 40:229-233.

96 Sparks, D.L., Martens, D.C., and Zelazny, L.W. 1980. Plant uptake and leaching of applied and indigenous potassium in Dothan soils. Agronomy Journal 72:551-555.

97 Woodruff, J.R. and Parks, C.L. 1980. Topsoil and subsoil potassium calibration with leaf potassium for fertility rating. Agronomy Journal 72:392-396.

98 Bray, R.H. 1940. The chemical nature of soil potassium in relation to its availability in Illinois soils. $\mathrm{PhD}$ thesis, University of Illinois, Urbana, IL.

99 Kuhlmann, H., Claassen, N., and Wehrmann, J. 1985. A method for determining the K-uptake from subsoil by plants. Plant and Soil 83:449-452.

100 Kuhlmann, H. 1990. Importance of the subsoil for the K nutrition of crops. Plant and Soil 127:129-136.

101 Witter, E. and Johansson, G. 2001. Potassium uptake from the subsoil by green manure crops. Biological Agriculture and Horticulture 19:127-141.

102 Jouany, C., Colomb, B., and Bosc, M. 1996. Long-term effects of potassium fertilization on yields and fertility status of calcareous soils of south-west France. European Journal of Agronomy 5:287-294.

103 Grimes, D.W. and Hanway, J.J. 1967. An evaluation of the availability of K in crop residues. Soil Science Society of America Proceedings 31:705-706.

104 Borisova, N.I., Vasineva, L.V., and Semenov, Y.I. 1984. Use of potassium from straw and green mass of barley, labelled with ${ }^{40} \mathrm{~K}$, by plants. Soviet Agricultural Science 12:28-30.

105 Dierolf, T.S. and Yost, R.S. 2000. Stover and potassium management in an upland rice-soybean rotation on an Indonesian Ultisol. Agronomy Journal 92:106-114.

106 Rosolem, C.A., Calonego, J.C., and Foloni, J.S.S. 2005. Potassium leaching from millet straw as affected by rainfall and potassium rates. Communications in Soil Science and Plant Analysis 36:1063-1074.

107 Glass, A.D.M. 1983. Regulation of ion transport. Annual Review of Plant Physiology 34:311-326.

108 Saha, M.N., Chaudhury, J., Saha, A.R., and Ray, P.K. 1998. Effect of intensive cropping and fertilizer use on soil properties and crop yields in a long-term experiment. In A. Swarup, D. Damodar Reddy and R.N. Prasad (eds). Proceedings of a National Workshop on Long-term Soil Fertility Management Integrated Plant Nutrient Supply. Indian Institute of Soil Science, Bhopal, India. p. 215-220. 
109 Walker, M.E., Flowers, R.A., Henning, R.J., Keisling, T.C., and Mullinix, B.G. 1979. Response of early bunch peanuts to calcium and potassium fertilization. Peanut Science 6:119-123.

110 Hartzog, D.L. and Adams, J.F. 1988. Relationship between soil-test $\mathrm{P}$ and $\mathrm{K}$ and yield response of runner peanuts to fertilizer. Communications in Soil Science and Plant Analysis 19:1645-1653.

111 Roberts, S. and Beaton, J.D. 1988. Potato Use of Phosphorus and Potassium in Sandy Soils. Research Bulletin XB1004. Washington State University, Pullman, WA.

112 Davenport, J.R. and Bentley, E.M. 2001. Does potassium fertilizer form, source, and time of application influence potato yield and quality in the Columbia Basin? American Journal of Potato Research 78:311-318.

113 Bailey, L.D. 1983. Effects of potassium fertilizer and fall harvests on alfalfa grown on the eastern Canadian prairies. Canadian Journal of Soil Science 63:211-219.

114 Rowberry, R.G. and Ketcheson, J.W. 1978. Sources of potassium in potato fertilizer and times of application. Canadian Journal of Plant Science 58:779-782.

115 Terman, G.L. 1977. Yields and nutrient accumulation by determinate soybeans, as affected by applied nutrients. Agronomy Journal 69:234-238.

116 Burmester, C.H. and Adams, F. 1983. Fertilizer requirements for new plantings of tall fescue and white clover on two low-fertility Ultisols. Agronomy Journal 75:936-940.

117 Laughlin, W.M., Martin, P.F., and Smith, G.R. 1973. Potassium rate and source influences on yield and composition of bromegrass forage. Agronomy Journal 65:85-87.

118 Bhangoo, M.S. and Albritton, D.J. 1972. Effect of fertilizer nitrogen, phosphorus, and potassium on yield and nutrient content of Lee soybeans. Agronomy Journal 64:743-746.

119 Maples, R. and Keogh, J.L. 1974. Fertilization of Cotton with Potassium, Magnesium, and Sulfur on Certain Delta Soils: Alfisols Formed from Mississippi River Valley Alluvium. Arkansas Agricultural Experiment Station Bulletin 787. University of Arkansas, Fayetteville, AR.

120 Rupe, J.C., Widick, J.D., Sabbe, W.E., Robbins, R.T., and Becton, C.B. 2000. Effect of chloride and soybean cultivar on yield and the development of sudden death syndrome, soybean cyst nematode, and southern blight. Plant Disease 84:669-674.

121 Cassman, K.G., Kerby, T.A., Roberts, B.A., Bryant, D.C., and Brouder, S.M. 1989. Differential response of two cotton cultivars to fertilizer and soil potassium. Agronomy Journal 81:870-876.

122 Linquist, B.A., Byous, E., Jones, G., Williams, J.F., Six, J., Horwath, W., and van Kessel, C. 2008. Nitrogen and potassium fertility impacts on aggregate sheath spot disease and yields of rice. Plant Production Science 11:260-267.

123 Barbarick, K.A. 1985. Potassium fertilization of alfalfa grown on a soil high in potassium. Agronomy Journal 77:442-445.

124 Allinson, D.W., Guillard, K., Rafey, M.M., Grabber, J.H., and Dest, W.M. 1992. Response of reed canarygrass to nitrogen and potassium fertilization. Journal of Production Agriculture 5:595-601.

125 Svec, L.V., Andrews, A.K., and Crittenden, H.W. 1976. Soybean (Glycine $\max$ (L.) Merr.) yield and disease incidence with potassium fertilization. Communications in Soil Science and Plant Analysis 7:727-741.

126 Sartain, J.B., Forbes, R.B., and Usherwood, N.R. 1979. Yield response of soybeans to $\mathrm{P}$ and $\mathrm{K}$ fertilization as correlated with soil extractable and tissue nutritional levels. Communications in Soil Science and Plant Analysis 10:1219-1232.

127 Elwali, A.M.O. and Gascho, G.J. 1983. Sugarcane response to $\mathrm{P}, \mathrm{K}$, and DRIS corrective treatments on Florida Histosols. Agronomy Journal 75:79-83.

128 Obreza, T.A. and Rhoads, F.M. 1988. Irrigated corn response to soil-test indices and fertilizer nitrogen, phosphorous, potassium, and magnesium. Soil Science Society of America Journal 52:701-706.

129 Welch, L.F., Adams, W.E., and Carmon, J.L. 1963. Yield response surfaces, isoquants, and economic fertilizer optima for coastal bermudagrass. Agronomy Journal 55:63-67.

130 Walker, M.E., Morris, H.D., and Carter, R.L. 1974. The Effect of Rate and Method of Application of N, P, and K on Yield, Quality and Chemical Composition of Spanish and Runner Peanuts. Georgia Agricultural Experiment Station Research Bulletin 152. University of Georgia, Athens, GA.

131 Parker, M.B., Gascho, G.J., and Gaines, T.P. 1983. Chloride toxicity of soybeans grown on Atlantic Coast Flatwoods soils. Agronomy Journal 75:439-443.

132 Walker, M.E., Gaines, T.P., and Parker, M.B. 1989. Potassium, magnesium, and irrigation effects on peanuts grown on two soils. Communications in Soil Science and Plant Analysis 20:1011-1032.

133 McDole, R.E., Stallknecht, G.F., Dwelle, R.B., and Pavek, J.J. 1978. Response of four potato varieties to potassium fertilization in a seed growing area of eastern Idaho. American Potato Journal 55:495-504.

134 Welch, L.F., Johnson, P.E., McKibben, G.E., Boone, L.V., and Pendleton, J.W. 1966. Relative efficiency of broadcast versus banded potassium for corn. Agronomy Journal 58:618-621.

135 Stivers, R.K. and Ohlrogge, A.J. 1952. Influence of phosphorus and potassium fertilization of two soil types on alfalfa yield, stand, and content of these elements. Agronomy Journal 44:618-621.

136 Hanway, J.J., Barber, S.A., Bray, R.H., Caldwell, A.C., Engelbert, L.E., Fox, R.L., Fried, M., Hovland, D., Ketcheson, J.W., Laughlin, W.M., Lawton, K., Lipps, R.C., Olson, R.A., Pesek, J.T., Pretty, K., Smith, F.W., and Stickney, E.M. 1961. North Central Regional Potassium Studies. I. Field Studies with Alfalfa. Iowa Agricultural Experiment Station Research Bulletin 494. Iowa State University, Ames, IA.

137 Sweeney, D.W., Moyer, J.L., Jardine, D.J., and Whitney, D.A. 2011. Nitrogen, phosphorus, and potassium effects on grain sorghum production and stalk rot following alfalfa and birdsfoot trefoil. Journal of Plant Nutrition 34:1330 1340 .

138 Templeton, W.C. Jr and Taylor, T.H. 1966. Yield response of a tall fescue-white clover sward to fertilization with nitrogen, phosphorus, and potassium. Agronomy Journal 58:319-322.

139 Gascho, G.J., Davis, J.F., Fogg, R.A., and Frakes, M.G. 1969. The effects of potassium carriers and levels of potassium and nitrogen fertilization on the yield and 
quality of sugar beets. Journal of the American Society of Sugar Beet Technologists 15:298-305.

140 Grissom, P. and Spurgeon, W.I. 1961. Fertility Practices for Cotton and Corn in the Yazoo-Mississippi Delta. Mississippi State University Agricultural Experiment Station Bulletin 614. Mississippi State University, State College, MS.

141 Pettigrew, W.T. 1999. Potassium deficiency increases specific leaf weights and leaf glucose levels in field-grown cotton. Agronomy Journal 91:962-968.

142 Adeli, A. and Varco, J.J. 2002. Potassium management effects on cotton yield, nutrition, and soil potassium level. Communications in Soil Science and Plant Analysis 25:2229-2242.

143 Pettigrew, W.T. 2003. Relationships between insufficient potassium and crop maturity in cotton. Agronomy Journal 95:1323-1329.

144 Wortmann, C.S., Dobermann, A.R., Ferguson, R.B., Hergert, G.W., Shapiro, C.A., Tarkalson, D.D., and Walters, D.T. 2009. High-yielding corn response to applied phosphorus, potassium, and sulfur in Nebraska. Agronomy Journal 101:546-555.

145 Malm, N.R. 1972. Fertilization of Established Alfalfa with Nitrogen and Potassium. New Mexico Agricultural Experiment Station Research Report 240. New Mexico State University, Las Cruces, NM.

146 Nelson, W.L., Burkhart, L., and Colwell, W.E. 1945. Fruit development, seed quality, chemical composition, and yield of soybeans as affected by potassium and magnesium. Soil Science Society of America Proceedings 10:224-229.

147 Goos, R.J., Johnson, B.E., and Holmes, B.M. 1987. Effect of potassium chloride fertilization on two barley cultivars differing in common root rot reaction. Canadian Journal of Plant Science 67:395-401.

148 Lueking, M.A., Johnson, J.W., and Himes, F.L. 1983. Effects of increasing the rates of potassium and nitrapyrin on nitrogen uptake by corn. Agronomy Journal 75:247-249.

149 Hudak, C., Stehouwer, R., and Johnson, J. 1989. An evaluation of $\mathrm{K}$ rate, placement and tillage systems for soybeans. Journal of Fertilizer Issues 6:25-31.

150 Robinson, R.R., Rhykerd, C.L., and Gross, C.F. 1962. Potassium uptake by orchardgrass as affected by time, frequency and rate of potassium fertilization. Agronomy Journal 54:351-353.

151 Alva, A.K. 1985. A comparison of fertilizer recommendations by soil testing program and alfalfa yield response in soils of two associations. Communications in Soil Science and Plant Analysis 16:213-228.

152 Gaspar, P.E., Reeves, D.L., Schumacher, T.E., and Fixen, P.E. 1994. Oat cultivar response to potassium chloride on soils testing high in potassium. Agronomy Journal 86:255-258.

153 Wittels, H. and Seatz, L.F. 1953. Effect of potash fertilization on yield, stalk breakage and mineral composition of corn. Soil Science Society of America Proceedings 17:369-371.

154 Clement-Bailey, J. and Gwathmey, C.O. 2007. Potassium effects on partitioning, yield, and earliness of contrasting cotton cultivars. Agronomy Journal 99:1130-1136.

155 Nelson, L.R., Keisling, T.C., and Rouquette, F.M. Jr 1983. Potassium rates and sources for coastal bermudagrass. Soil Science Society of America Journal 47:963-966.
156 Westermann, D.T., Tindall, T.A., James, D.W., and Hurst, R.L. 1994. Nitrogen and potassium fertilization of potatoes: Yield and specific gravity. American Potato Journal 71:417-431.

157 Camper, H.M. and Lutz, J.A. Jr 1977. Plowsole placement of fertilizer for soybeans and response to tillage of plowsole. Agronomy Journal 69:701-704.

158 Jones, G.D., Lutz, J.A. Jr, and Smith, T.J. 1977. Effects of phosphorus and potassium on soybean nodules and seed yield. Agronomy Journal 69:1003-1006.

159 Smith, D. 1975. Effects of potassium topdressing a low fertility silt loam soil on alfalfa herbage yields and composition and on soil $\mathrm{K}$ values. Agronomy Journal 67:60-64.

160 Rominger, R.S., Smith, D., and Peterson, L.A. 1976. Yield and chemical composition of alfalfa as influenced by high rates of $\mathrm{K}$ topdressed as $\mathrm{KCl}$ and $\mathrm{K}_{2} \mathrm{SO}_{4}$. Agronomy Journal 68:573-577.

161 Smith, D. and Smith, R.R. 1977. Responses of red clover to increasing rates of topdressed potassium fertilizer. Agronomy Journal 69:45-48.

162 Smith, D. and Powell, R.D. 1979. Yield of alfalfa as influenced by levels of $\mathrm{P}$ and $\mathrm{K}$ fertilization. Communications in Soil Science and Plant Analysis 10:531-543.

163 Panique, E., Kelling, K.A., Schulte, E.E., Hero, D.E., Stevenson, W.R., and James, R.V. 1997. Potassium rate and source effects on potato yield, quality, and disease interaction. American Potato Journal 74:379-398.

164 Fageria, N.K., Wright, R.J., Baligar, V.C., and Carvalho, J.R.P. 1990. Upland rice response to potassium fertilization on a Brazilian Oxisol. Fertilizer Research 21:141-147.

165 Rosolem, C.A. and Nakagawa, J. 2001. Residual and annual potassic fertilization for soybeans. Nutrient Cycling in Agroecosystems 59:143-149.

166 Cox, F.R. and Uribe, E. 1992. Management and dynamics of potassium in a humid tropical Ultisol under a ricecowpea rotation. Agronomy Journal 84:655-660.

167 Casanova, E. 2000. Phosphorus and potassium fertilization and mineral nutrition of soybean. Interciencia 25:92-95.

168 Jekić, M., Trpeski, V., and Raimi, N. 1998. The effect of $\mathrm{KCl}$ and $\mathrm{K}_{2} \mathrm{SO}_{4}$ in NPK fertilizers on yield, quality and economical earning by production of potato on alluvium of the Vardar. Macedonian Agricultural Review 45:11-15.

169 Gaydou, E.M. and Arrivets, J. 1983. Effects of phosphorus, potassium, dolomite, and nitrogen fertilization on the quality of soybean. Yields, proteins, and lipids. Journal of Agricultural and Food Chemistry 31:765-769.

170 Lombin, G. and Mustafa, S. 1981. Potassium response of cotton on some Inceptisols and Oxisols of northern Nigeria. Agronomy Journal 73:724-729.

171 Lombin, G. and Singh, L. 1986. Fertilizer responses of groundnuts (Arachis hypogae L.) under continuous intensive cultivation in the Nigerian savannah. Fertilizer Research 10:43-58.

172 Rahman, M.A., Chakravarty, D., Shahidullah, M., and Hossain, M.T. 1978. Studies on the effect of nitrogen phosphorus and potassium on the growth, yield and nutrient contents of safflower (Carthamus tinctorius L.). Bangladesh Journal of Scientific and Industrial Research 13:32-39. 
173 Chowdhury, M.K.A. and Rahman, M.H. 1990. Potash requirement of sugarcane in Gangetic River flood plain soils of Bangladesh. Journal of the Indian Society of Soil Science 38:688-691.

174 Bokhtiar, S.M., Karim, A.J.M.S., Hossain, K.M., Hossain, T., and Egashira, K. 2001. Response of radish to varying levels of irrigation water and fertilizer potassium on clay terrace soil of Bangladesh. Communications in Soil Science and Plant Analysis 32:2979-2991.

175 Hymowitz, T., Jethmalani, S.C., Tiwari, K.L., and Walker, W.M. 1971. Effect of inoculum, variety, nitrogen, phosphorus, and potassium on yield, protein and oil content of soybeans at Jabalpur, M. P. India. Communications in Soil Science and Plant Analysis 2:283-292.

176 Gupta, B.R., Pathak, R.K., Bhan, S., and Singh, A. 1972. Effect of NPK on yield, nutrient uptake and quality of toria (Brassica campestris Var. Toria T.9). Indian Journal of Agronomy 17:88-91.

177 Stilwell, T.C., Sekhon, G.S., and Arscott, T.G. 1975. Response to potassium fertilization of three Punjab soils. Indian Journal of Agricultural Science 45:149-151.

178 Grewal, J.S. and Singh, S.N. 1980. Effect of potassium nutrition on frost damage and yield of potato plants on alluvial soils of the Punjab (India). Plant and Soil 57:105-110.

179 Mehta, S.C., Singh, N., Mittal, S.B., and Singh, M. 1991. Response of paddy and wheat to nitrogen and potassium fertilization. Crop Research 4:84-89.

180 Subramanian, K.S. 1994. Influence of soil and foliar application of potassium on growth, nutrient utilization, yield and quality of sugarcane (Saccharum officinarum L.). New Botanist 21:13-20.

181 Singh, K.N. and Sharma, D.P. 2001. Response of wheat to nitrogen and potassium in saline soils. Experimental Agriculture 37:417-427.

182 Kumar, P., Pandey, S.K., Singh, S.V., Rawal, S., and Kumar, D. 2004. Effect of potassium fertilization on processing grade tuber yield and quality parameters in potato (Solanum tuberosum). Indian Journal of Agricultural Science 74:177-179.

183 Moinuddin, Singh, K., Bansal, S.K., and Pasricha, N.S. 2004. Influence of graded levels of potassium fertilizer on growth, yield, and economic parameters of potato. Journal of Plant Nutrition 27:239-259.

184 Gunarto, L., Yahya, M., Supadmo, H., and Buntan, A. 1985. Response of corn to NPK fertilization grown in a latosol in South Salawesi, Indionesia. Communications in Soil Science and Plant Analysis 16:1179-1188.

185 Hanolo, W. and Pulung, M.A. 1994. Effect of P and K fertilizers on growth and yield of pea. Acta Horticulturae 369:335-339.

186 Bakhsh, A., Khattak, J.K., and Bhatti, A.U. 1986. Comparative effect of potassium chloride and potassium sulfate on the yield and protein content of wheat in three different rotations. Plant and Soil 96:273-277.

187 Khan, Q., Himayatullah, M.N., Khan, N., and Shah, Z. 1994. Effect of various levels of potassium on wheat crop. Sarhad Journal of Agriculture 10:337-343.

188 Gormus, O. 2002. Effects of rate and time of potassium application on cotton yield and quality in Turkey. Journal of Agronomy and Crop Science 188:382-388.

189 Nguyen, H., Schoenau, J.J., Nguyen, D., Van Rees, K., and Boehm, M. 2002. Effects of long-term nitrogen, phosphorus, and potassium fertilization on cassava yield and plant nutrient composition in North Vietnam. Journal of Plant Nutrition 25:425-442.

190 Brennan, R.F. and Bolland, M.D.A. 2007. Influence of potassium and nitrogen fertiliser on yield, oil and protein concentration of canola (Brassica napus L.) grain harvested in south-western Australia. Australian Journal of Experimental Agriculture 47:976-983.

191 Hall, A.M. 1975. Effect of phosphorus, potassium, and calcium on peanuts at Mauke (Cook Islands). New Zealand Journal of Experimental Agriculture 3:117-120.

192 Rehm, G.W., Sorensen, R.C., and Wiese, R.A. 1981. Application of phosphorus, potassium, and zinc to corn grown for grain or silage: Early growth and yield. Soil Science Society of America Journal 45:523528.

193 Hoeft, R.G., Walsh, L.M., and Liegel, E.A. 1975. Effect of seed-placed fertilizer on the emergence (germination) of soybeans (Glycine max L.) and snapbeans (Phaseolus vulgaris L.). Communications in Soil Science and Plant Analysis 6:655-664.

194 Mascarenhas, H.A.A., Hiroce, R., and Braga, N.R. 1976. Cloreto de potassio para a soja. Bragantia 35:CXXVCXXVII.

195 Sangoi, L., Ernani, P.R., Bianchet, P., Vargas, V.P., and Picoli, G.J. 2009. Efeito de doses de cloreto de potássio sobre a germinação e o crescimento inicial do milho, em solos com texturas contrastantes. Revista Brasileira de Milho e Sorgo 8:187-197.

196 Curtin, D., Steppuhn, H., Campbell, C.A., and Biederbeck, V.O. 1999. Carbon and nitrogen mineralization in soil treated with chloride and phosphate salts. Canadian Journal of Soil Science 79:427-429.

197 Roseberg, R.J., Christensen, N.W., and Jackson, T.L. 1986. Chloride, soil solution osmotic potential, and soil $\mathrm{pH}$ effects on nitrification. Soil Science Society of America Journal 50:941-945.

198 Smith, D. and Peterson, L.A. 1975. Chlorine concentrations in alfalfa herbage and soil with $\mathrm{KCl}$ topdressing of a low fertility silt loam soil. Communications in Soil Science and Plant Analysis 6:521-533.

199 Hahn, B.E., Olson, F.R., and Roberts, J.L. 1942. Influence of potassium chloride on nitrification in Bedford silt loam. Soil Science 54:113-121.

200 Golden, D.C., Sivasubramaniam, S., Sandanam, S., and Wijedasa, M.A. 1984. Inhibitory effects of commercial potassium chloride on the nitrification rates of added ammonium sulphate in an acid red yellow podzolic soil. Tea Quarterly 53:43-46.

201 Younts, S.E. and Musgrave, R.B. 1958. Chemical composition, nutrient absorption, and stalk rot incidence of corn as affected by chloride in potassium fertilizer. Agronomy Journal 50:426-429.

202 Timm, C.A., Goos, R.J., Johnson, B.E., Sobolik, F.J., and Stack, R.W. 1986. Effect of potassium fertilizers on malting barley infected with common root rot. Agronomy Journal 78:197-200.

203 Johnston, A.E., Goulding, K.W.T., and Mercer, E. 1993. Potassium leaching from a sandy loam soil. Potash Review 12(4):1-16.

204 Askegaard, M. and Eriksen, J. 2000. Potassium retention and leaching in an organic crop rotation on loamy sand as 
affected by contrasting potassium budgets. Soil Use and Management 16:200-205.

205 Brye, K.R. and Norman, J.M. 2004. Land-use effects on anion-associated cation leaching in response to above-normal precipitation. Acta Hydrochimica et Hydrobiologica 32:235-248.

206 Karamanos, R.E., Harapiak, J.T., and Flore, N.A. 2003. Application of seed-row potash to barley (Hordeum vulgare L.) grown on soils with high "available" potassium levels. Canadian Journal of Plant Science 83:291-303.

207 Brennan, R.F. and Jayasena, K.W. 2007. Increasing applications of potassium fertiliser to barley crops grown on deficient sandy soils increased grain yields while decreasing some foliar diseases. Australian Journal of Agricultural Research 58:680-689.

208 Grant, C.A., Bailey, L.D., and Therrien, M.C. 1996. The effect of $\mathrm{N}, \mathrm{P}$ and $\mathrm{KCl}$ fertilizers on grain yield and $\mathrm{Cd}$ concentration of malting barley. Fertilizer Research 45:153-161.

209 Zubriski, J.C., Vasey, E.H., and Norum, E.B. 1970. Influence of nitrogen and potassium fertilizers and date of seeding on yield and quality of malting barley. Agronomy Journal 62:216-219.

210 Tinline, R.D., Ukrainetz, H., and Spurr, D.T. 1993. Effect of fertilizers and of liming acid soil on common root rot in wheat, and of chloride on the disease in wheat and barley. Canadian Journal of Plant Pathology 15:65-73.

211 Walker, W.M. and Raines, G.A. 1988. Effect of corn cultivar, phosphorus and potassium on yield and chemical composition. Journal of Plant Nutrition 11:1715-1726.

212 Heckman, J.R. 1998. Corn stalk rot suppression and grain yield response to chloride. Journal of Plant Nutrition 21:149-155.

213 Ebelhar, S.A., Kamprath, E.J., and Moll, R.H. 1987. Effects of nitrogen and potassium on growth and cation composition of corn genotypes differing in average ear number. Agronomy Journal 79:875-881.

214 Fintel, R.G.H. von and Quicke, G.V. 1962. Soil fertilization and the protein, zein, essential amino acid and niacin content of maize grain. South African Journal of Agricultural Science 5:165-175.

215 Boswell, F.C. and Parks, W.L. 1957. The effect of soil potassium levels on yield, lodging, and mineral composition of corn. Soil Science Society of America Proceedings 21:301-305.

216 Liebhardt, W.C. and Murdock, J.T. 1965. Effect of potassium on morphology and lodging of corn. Agronomy Journal 57:325-328.

217 Haq, M.U. and Mallarino, A.P. 2005. Response of soybean grain oil and protein concentrations to foliar and soil fertilization. Agronomy Journal 97:910-918.

218 Helz, G.E. and Whiting, A.L. 1928. Effects of fertilizer treatment on the formation of nodules on the soybean. Journal of the American Society of Agronomy 20:975-981.

219 Sharma, P., Duveiller, E., and Sharma, R.C. 2006. Effect of mineral nutrients on spot blotch severity in wheat, and associated increases in grain yield. Field Crops Research 95:426-430.

220 Norvell, W.A., Wu, J., Hopkins, D.G., and Welch, R.M. 2000. Association of cadmium in durum wheat grain with soil chloride and chelate-extractable soil cadmium. Soil Science Society of America Journal 64:2162-2168.
221 Wahhab, A. and Ali, R. 1962. Effect of nitrogen, phosphorus and potash on lodging, yield, plant characters and the chemical composition of irrigated wheat. Pakistan Journal of Scientific Research 14:8-16.

222 Bennett, O.L., Rouse, R.D., Ashley, D.A., and Doss, B.D. 1965. Yield, fiber quality and potassium content of irrigated cotton plants as affected by rates of potassium. Agronomy Journal 57:296-299.

223 Bernardi, A.C. de C., Oliveira, J.P. de Jr, Leandro, W.M., Mesquita, T.G. da S., Freitas, P.L. de, and Carvalho, M. da C.S. 2009. Doses e formas de aplicação da adubação potássica na rotação soja, milheto e algodão em sistema plantio direto. Pesquisa Agropecuária Tropical 39:158-167.

224 Cassman, K.G., Kerby, T.A., Roberts, B.A., Bryant, D.C., and Higashi, S.L. 1990. Potassium nutrition effects on lint yield and fiber quality of Acala cotton. Crop Science 30:672-677.

225 Minton, E.B. and Ebelhar, M.W. 1991. Potassium and aldicarb-disulfoton effects on verticillium wilt, yield, and quality of cotton. Crop Science 31:209-212.

226 Pettigrew, W.T., Heitholt, J.J., and Meredith, W.R. Jr 1996. Genotypic interactions with potassium and nitrogen in cotton of varied maturity. Agronomy Journal 88:89-93.

227 Pettigrew, W.T., Meredith, W.R. Jr, and Young, L.D. 2005. Potassium fertilization effects on cotton lint yield, yield components, and reniform nematode populations. Agronomy Journal 97:1245-1251.

228 Peters, J.B., Kelling, K.A., Speth, P.E., and Offer, S.M. 2005. Alfalfa yield and nutrient uptake as affected by $\mathrm{pH}$ and applied K. Communications in Soil Science and Plant Analysis 36:583-596.

229 Rogers, G., Porter, R., Jolley, L.C., and Leaver, D.D. 1977. Management factors and grass tetany in dairy cattle. Australian Veterinary Journal 53:523-528.

230 Ketterings, Q.M., Godwin, G., Cherney, J.H., and Kilcer, T.F. 2005. Potassium management for brown midrib sorghum $\times$ sudangrass as replacement for corn silage in the north-eastern USA. Journal of Agronomy and Crop Science 191:41-46.

231 Meissner, A.P. and Clarke, A.L. 1977. Response of mown pasture to potassium fertilizer in south-eastern South Australia. Australian Journal of Experimental Agriculture and Animal Husbandry 17:765-775.

232 Balasko, J.A. 1977. Effects of N, P, and K fertilization on yield and quality of tall fescue forage in winter. Agronomy Journal 69:425-428.

233 Brennan, R.F. and Bolland, M.D.A. 2006. Soil and tissue tests to predict the potassium requirements of canola in south-western Australia. Australian Journal of Experimental Agriculture 46:675-679.

234 Li, Y.-M., Chaney, R.L., and Schneiter, A.A. 1994. Effect of soil chloride level on cadmium concentration in sunflower kernels. Plant and Soil 167:275-280.

235 Tinker, P.B.H. 1965. The effects of nitrogen, potassium and sodium fertilizers on sugar beet. Journal of Agricultural Science, Cambridge 65:207-212.

236 Otto, R., Vitti, G.C., and de Cerqueira Luz, P.H. 2010. Manejo da adubação potássica na cultura da cana-deaçúcar. Revista Brasileira de Ciencia do Solo 34:1137-1145.

237 Gascho, G.J. and Kidder, G. 1979. Responses to Phosphorus and Potassium and Fertilizer Recommendations for Sugarcane in South Florida. 
Florida Agricultural Experimental Station Bulletin 809. University of Florida, Gainesville, FL.

238 Maier, N.A., Dahlenburg, A.P., and Frensham, A.B. 1986. Potassium nutrition of irrigated potatoes in South Australia. 3. Effect on specific gravity, size and internal bruising of tubers. Australian Journal of Experimental Agriculture 26:737-744.

239 Sparrow, L.A., Salardini, A.A., and Johnstone, J. 1994. Field studies of cadmium in potatoes (Solanum tuberosum L.). III. Response of cv. Russet Burbank to sources of banded potassium. Australian Journal of Agricultural Research 45:243-249.

240 McLaughlin, M.J., Maier, N.A., Freeman, K., Tiller, K. G., Williams, C.M.J., and Smart, M.K. 1995. Effect of potassic and phosphatic fertilizer type, fertilizer $\mathrm{Cd}$ concentration and zinc rate on cadmium uptake by potatoes. Fertilizer Research 40:63-70.

241 Allison, M.F., Fowler, J.H., and Allen, E.J. 2001. Responses of potato (Solanum tuberosum) to potassium fertilizers. Journal of Agricultural Science, Cambridge 136:407-426.

242 McDole, R.E. 1978. Potassium fertilizer trials with potatoes on coarse-textured soils in southeastern Idaho. American Potato Journal 55:161-170.

243 Herlihy, M. and Carroll, P.J. 1969. Effects of N, P and K and their interactions on yield, tuber blight and quality of potatoes. Journal of the Science of Food and Agriculture 20:513-517.

244 Murphy, H.J. and Goven, M.J. 1959. Factors Affecting the Specific Gravity of the White Potato in Maine. Maine Agricultural Experiment Station Bulletin 583. University of Maine, Orono, ME.

245 Hahlin, M. and Johansson, O. 1973. Fosfor- och kaliumgödsling till matpotatis. Lantbruks-högskolans meddelande. Serie A. NR 192. Royal Agricultural College of Sweden, Uppsala, Sweden.

246 Constantin, R.J., Jones, L.G., and Hernandez, T.P. 1977. Effects of potassium and phosphorus fertilization on quality of sweet potatoes. Journal of the American Society of Horticultural Science 102:779-781.

247 McLaughlin, M.J., Palmer, L.T., Tiller, K.G., Beech, T.A., and Smart, M.K. 1994. Increased soil salinity causes elevated cadmium concentrations in field-grown potato tubers. Journal of Environmental Quality 23:10131018.

248 Julin, B., Wolk, A., Bergkvist, L., Bottai, M., and Åkesson, A. 2012. Dietary cadmium exposure and risk of postmenopausal breast cancer: A population-based prospective cohort study. Cancer Research 72:1459-1466.

249 Goff, J.P. and Horst, R.L. 1997. Effects of addition of potassium or sodium, but not calcium, to prepartum rations on milk fever in dairy cows. Journal of Dairy Science 80:176-186.

250 Horst, R.L., Goff, J.P., Reinhardt, T.A., and Buxton, D.R. 1997. Strategies for preventing milk fever in dairy cattle. Journal of Dairy Science 80:1269-1280.

251 Allcroft, R. and Burns, K.N. 1968. Hypomagnesaemia in cattle. New Zealand Veterinary Journal 16:109-128.

252 Cherney, J.H., Cherney, D.J.R., and Bruulsema, T.W. 1998. Potassium management. In J.H. Cherney and D.J.R. Cherney (eds). Grass for Dairy Cattle. CAB International, Wallingford, England. p. 137-160.
253 McCalla, T.M. 1937. Behavior of Legume Bacteria (Rhizobium) in Relation to Exchangeable Calcium and Hydrogen Ion Concentration of the Colloidal Fraction of the Soil. Missouri Agricultural Experiment Station Research Bulletin 256. University of Missouri, Columbia, MO.

254 Albrecht, W.A. and Davis, F.L. 1929. Physiological importance of calcium in legume inoculation. Botanical Gazette 88:310-321.

255 Heaney, R.P. and Barger-Lux, M.J. 1994. Low calcium intake: The culprit in many chronic diseases. Journal of Dairy Science 77:1155-1160.

$256 \mathrm{Wu}$, K., Willett, W.C., Fuchs, C.S., Colditz, G.A., and Giovannucci, E.L. 2002. Calcium intake and risk of colon cancer in women and men. Journal of the National Cancer Institute 94:437-446.

257 Newmark, H.L., Heaney, R.P., and Lachance, P.A. 2004. Should calcium and vitamin $\mathrm{D}$ be added to the current enrichment program for cereal-grain products? American Journal of Clinical Nutrition 80:264-270.

258 Nordin, B.E.C. 1997. Calcium and osteoporosis. Nutrition 13:664-686.

259 Centeno, V., de Barboza, G.D., Marchionatti, A., Rodríguez, V., and de Talamoni, N.T. 2009. Molecular mechanisms triggered by low-calcium diets. Nutrition Research Reviews 22:163-174.

260 Krauss, A. and Saurat, A. 1989. Potassium sulphate: K fertilizers for arid and semi-arid zones. In Proceedings of the Workshop on the Role of Potassium in Improving Fertilizer Use Efficiency. National Fertilizer Development Corporation, Islamabad, Pakistan. p. 171185.

261 Lucas, R.E., Wheeler, E.J., and Davis, J.F. 1954. Effect of potassium carriers and phosphate:potash ratios on the yield and quality of potatoes grown in organic soils. American Potato Journal 31:349-352.

262 Dickins, J.C., Harrap, F.E.G., and Holmes, M.R.J. 1962. Field experiments comparing the effects of muriate and sulphate of potash on potato yield and quality. Journal of Agricultural Science, Cambridge 59:319-326.

263 Rowberry, R.G., Sherrell, C.G., and Johnston, G.R. 1963. Influence of rates of fertilizer and sources of potassium on the yield, specific gravity and cooking quality of Katahdin potatoes. American Potato Journal 40:177-181.

264 Lujan, L. and Smith, O. 1964. Potato quality XXV. Specific gravity and after-cooking darkening of Katahdin potatoes as influenced by fertilizers. American Potato Journal 41:274-278.

265 Højmark, J.V. 1977. Virkningen af stigende mængde kaliumklorid og kaliumsulfat på kartoffeludbytte, kogekvalitet og farve af chips. Tiddskrift for Planteavl 81:68-80.

266 Rosolem, C.A., Nakagawa, J., Machado, J.R., and Yamada, T. 1979. Efeitos de modos de aplicação, doses e fontes de potássio na produção da soja. Revista de Agricultura, Piracicaba 54:13-19.

267 Abd El-Hadi, A.H., Khadr, M.S., El-Kholy, M.H., Zahran, F.A., and Negm, A.Y. 2002. Comparative effect of potassium sulphate and potassium chloride on crop production and soil chemical properties under Egyptian conditions. Egyptian Journal of Agricultural Research 80:521-537. 
268 Bansil, P.C. 2004. Agricultural subsidies. A global view. In B. Dorin and T. Jullien (eds). Agricultural Incentives in India. Past Trends and Prospective Paths Towards Sustainable Development. Manohar Publishers, New Delhi, India. p. 39-65.

269 Environmental Working Group. 2012. Farm Subsidy Database. Available at Web site http://farm.ewg.org/ (verified May 25, 2012).

270 Brown, W.W. 1950-1963. Commercial Fertilizer Year Book. Walter W. Brown, Atlanta, GA.

271 Illinois Department of Agriculture. 1964-2010. Illinois Commercial Fertilizer Tonnage Report. Illinois Department of Agriculture, Springfield, IL. Available since 2004 at Web site http://www.agr.state.il.us/programs/ fert2/ (verified August 9, 2012).

272 United States Department of Agriculture. 2012. USDA National Agricultural Statistics Service. Available at Web site http://www.nass.usda.gov (verified May 25, 2012).

273 Tributh, H., Boguslawski, E.v., Lieres, A.v., Steffens, D., and Mengel, K. 1987. Effect of potassium removal by crops on transformation of illitic clay minerals. Soil Science 143:404-409.

274 Frydman, S., Ravina, E., and Ehrenreich, T. 1977. Stabilization of heavy clay with potassium chloride. Geotechnical Engineering 8:95-108.

275 Abood, T.T., Kasa, A.B., and Chik, Z.B. 2007. Stabilisation of silty clay soil using chloride compounds. Journal of Engineering Science and Technology 2:102-110. Available at Web site http://jestec.taylors.edu.my/Vol\%
202\%20Issue \%201\%20April \%2007/102-110\%20Abood.pdf (verified August 9, 2012).

276 Chaitanya G, K.N.K., Prasada Raju, G.V.R., and Ramu, K. 2011. Evaluation studies of expansive soil treated with electrolytes. International Journal of Engineering Science and Technology 3:8298-8306. Available at Web site http://www.idc-online.com/technical_references/pdfs/ civil_engineering/EVALUATION\%20STUDIES $\% 20 \mathrm{OF}$

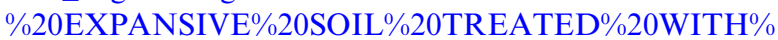
20ELECTROLYTES.pdf (verified August 9, 2012).

277 Venkara Muthyalu, P., Ramu, K., and Prasada Raju, G.V.R. 2012. Study on performance of chemically stabilized expansive soil. International Journal of Advances in Engineering and Technology 2:139-148. Available at Web site http://www.archives-ijaet.org/media/15I6IJAET0612724.pdf (verified August 9, 2012).

278 Deist, J. and Talibudeen, O. 1967. Ion exchange in soils from the ion pairs K-Ca, K-Rb, and K-Na. Journal of Soil Science 18:125-137.

279 Palaniappan, S., Peck, T.R., and Beavers, A.H. 1973. Effect of application of high rates of potassium on cation exchange capacity and potassium status of two Illinois soils. Communications in Soil Science and Plant Analysis 4:31-40.

280 Cassman, K.G., Roberts, B.A., Kerby, T.A., Bryant, D.C., and Higashi, S.L. 1989. Soil potassium balance and cumulative cotton response to annual potassium additions on a vermiculitic soil. Soil Science Society of America Journal 53:805-812. 